\title{
Quantum Brownian motion of a particle from Casimir-Polder interactions
}

\author{
Kanupriya Sinha $\circledast^{1,2, *}$ and Yiğit Subaşı $\oplus^{3, \dagger}$ \\ ${ }^{1}$ U.S. Army Research Laboratory, Adelphi, Maryland 20783, USA \\ ${ }^{2}$ Joint Quantum Institute, University of Maryland, College Park, Maryland 20742, USA \\ ${ }^{3}$ Computer, Computational and Statistical Sciences Division, Los Alamos National Laboratory, Los Alamos, New Mexico 87545, USA
}

(Received 7 October 2019; revised manuscript received 21 February 2020; accepted 21 February 2020; published 13 March 2020)

\begin{abstract}
We study the fluctuation-induced dissipative dynamics of the quantized center-of-mass motion of a polarizable dielectric particle trapped near a surface. The particle's center of mass is treated as an open quantum system coupled to the electromagnetic field acting as its environment, with the resulting system dynamics described by a quantum Brownian motion master equation. The dissipation and decoherence of the particle's center of mass are characterized by the modified spectral density of the electromagnetic field that depends on surface losses and the strength of the classical trap field. Our results are relevant to experiments with levitated dielectric particles near surfaces, illustrating potential ways of mitigating fluctuation-induced decoherence while preparing such systems in macroscopic quantum states.
\end{abstract}

DOI: 10.1103/PhysRevA.101.032507

\section{INTRODUCTION}

Creating macroscopic superpositions of massive systems as a means to understand the quantum-to-classical transition is a task of foundational importance [1]. Among the promising experimental platforms for realizing large superpositions of massive objects, levitated optomechanical systems bring together the advantages of optical trapping and cooling methods in terms of control, while being well isolated from an environment in the absence of mechanical clamping, thus minimizing decoherence [2-6]. There has been astonishing experimental progress in terms of the control and manipulation of levitated dielectric nanoparticles-ranging from recent demonstrations of cooling particles down to micro- and millidegrees Kelvin [7-9], to the observation of rotational frequencies as large as $\mathrm{MHz}-\mathrm{GHz}$ with remarkable stabilities [10-12].

Interfacing such precisely controlled mesoscopic quantum systems with waveguides further allows for better manipulation and probing mechanisms of the system of interest, as guided photonic modes can couple efficiently to particles in the near-field regime [13-15]. Near-field levitated nanophotonics can therefore allow for strong optomechanical couplings of mesoscopic systems with well-controlled fields, as has been demonstrated in Ref. [16].

However, when preparing a system in a macroscopic quantum state near surfaces, one needs to consider that the quantum (and thermal) fluctuations of the electromagnetic (EM)

\footnotetext{
*kanu@umd.edu

†ysubasi@lanl.gov

Published by the American Physical Society under the terms of the Creative Commons Attribution 4.0 International license. Further distribution of this work must maintain attribution to the author(s) and the published article's title, journal citation, and DOI.
}

field are enhanced due to the presence of the surface degrees of freedom [17]. The increased density of EM field modes can therefore cause the system of interest to decohere faster in the vicinity of a surface, as has been shown both theoretically and experimentally with regard to the internal degrees of freedom of particles near surfaces [18-24]. It is similarly imperative to analyze the fluctuation-induced decoherence for the external degrees of freedom in near-field nanomechanical experiments [25].

In this paper we study the decoherence and dissipation of the quantized center-of-mass (c.m.) motion of a neutral dielectric particle trapped near a surface. We show that the open system dynamics of the particle can be described in terms of the quantum Brownian motion (QBM) master equation [26-28], and the surface-modified dissipation and decoherence can be expressed in terms of a modified spectral density of the electromagnetic field. We further draw a correspondence between the surface-induced decoherence of the particle's c.m. and the collisional model of decoherence $[29,30]$.

The paper is organized as follows. In Sec. II we develop a theoretical model of a polarizable dielectric particle interacting with the EM field in the presence of a surface, deriving the fluctuation- and drive-induced potentials. In Sec. III we derive the QBM master equation for the quantized c.m. motion, and analyze the resulting decoherence and dissipation of the particle for different surface properties in Sec. IV. We summarize our findings in Sec. V.

\section{MODEL}

Let us consider a dielectric particle of mass $M$ and polarizability $\overline{\bar{\alpha}}(\omega)$ placed at a distance $z$ from a planar half-space medium of permittivity $\epsilon_{S}(\omega)$, as depicted in Fig. 1(a). We consider a classical driving field that is incident normally on the surface of the medium and reflected to form a standingwave potential. We assume that the particle is trapped near the 


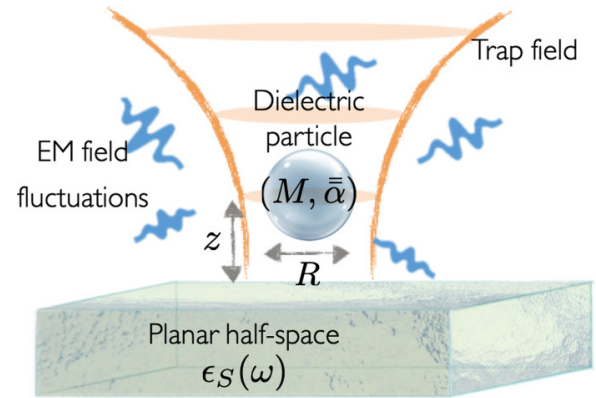

(a)
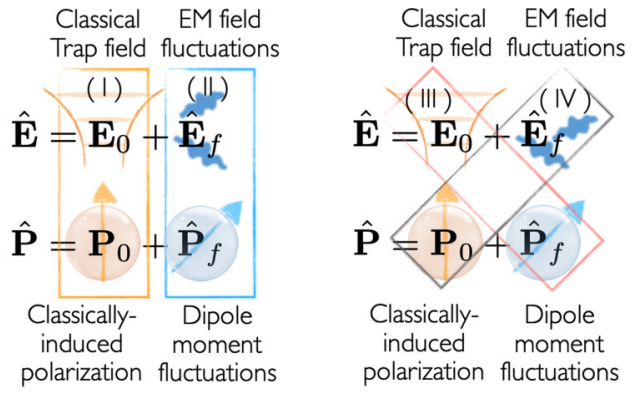

(b)

FIG. 1. (a) Schematic representation of a dielectric particle of mass $M$, radius $R$, and polarizability $\overline{\bar{\alpha}}(\omega)$ near a planar half space with permittivity $\epsilon_{S}(\omega)$, interacting with the quantum fluctuations of the EM field. We assume a classical trap field incident normally on the surface which creates a standing-wave potential perpendicular to the surface. The particle is trapped in the first intensity maxima of the standing-wave potential, at a distance $z$ from the surface. (b) The four contributions to the total interaction energy as a result of the interaction between the total electric field and the induced polarization of the particle: (I) corresponds to the classical trap potential $\hat{H}_{\mathrm{Tr}}$ due to the interaction of the trap field with the classically induced polarization [Eq. (8)]. (II) stands for the Casimir-Polder interaction $\hat{H}_{\mathrm{CP}}$ arising from the interaction between the fluctuations of the EM field with the fluctuating dipole moment of the particle [Eq. (9)]. (III) and (IV) taken together lead to the drive-induced Casimir-Polder Hamiltonian $\hat{H}_{\mathrm{DCP}}$ [Eq. (10)]. (III) arises from the interaction between the classical field and the dipole moment fluctuations and (IV) corresponds to the interaction between the classically induced polarization and the EM field fluctuations.

first intensity maxima of the standing-wave potential near the surface [16].

The Hamiltonian for the total system can be written as

$$
\hat{H}=\frac{\hat{p}_{z}^{2}}{2 m}+\hat{H}_{F}+\hat{H}_{\mathrm{int}},
$$

where the first term corresponds to the kinetic energy of the particle with $\hat{p}_{z}$ as the quantized c.m. momentum along the $z$ axis. In the absence of a strong transverse confinement we ignore the quantized motion in the $x y$ plane. $\hat{H}_{F}$ corresponds to the Hamiltonian of the quantized field in the presence of the medium [see Eq. (A1)]. The interaction Hamiltonian $\hat{H}_{\text {int }}$ represents the electric-dipole interaction between the polarizable particle and the total electric field over the volume $V$ of the particle given by

$$
\hat{H}_{\mathrm{int}}=-\int_{V} d^{3} r^{\prime} \hat{\mathbf{P}}\left(\mathbf{r}^{\prime}\right) \cdot \hat{\mathbf{E}}\left(\mathbf{r}^{\prime}\right)
$$

where $\hat{\mathbf{P}}\left(\mathbf{r}^{\prime}\right)$ refers to the polarization of the dielectric, and $\hat{\mathbf{E}}\left(\mathbf{r}^{\prime}\right)$ is the electric field, with $\mathbf{r}^{\prime}$ being a point in the volume of the particle.

The total electric field at a position $\mathbf{r}$ can be expressed as $\hat{\mathbf{E}}\left(\mathbf{r}^{\prime}\right)=\mathbf{E}_{0}\left(\mathbf{r}^{\prime}, t\right)+\hat{\mathbf{E}}_{f}\left(\mathbf{r}^{\prime}\right)$, where $\mathbf{E}_{0}\left(\mathbf{r}^{\prime}\right)$ is the classical trap field, and $\hat{\mathbf{E}}_{f}\left(\mathbf{r}^{\prime}\right)$ refers to the fluctuations of the field (quantum and thermal). We assume that the trap field is given as

$$
\mathbf{E}_{0}\left(\mathbf{r}^{\prime}, t\right)=\frac{1}{2}\left[\mathcal{E}_{0}\left(\mathbf{r}^{\prime}\right) e^{-i \omega_{0} t}+\mathcal{E}_{0}^{*}\left(\mathbf{r}^{\prime}\right) e^{i \omega_{0} t}\right],
$$

with $\omega_{0}$ as the frequency, and $\mathcal{E}_{0}\left(\mathbf{r}^{\prime}\right)$ as the amplitude of the electric field at position $\mathbf{r}^{\prime}$ which takes the incident and reflected fields into consideration. We further assume that the field is polarized along the $x y$ plane.

Using the macroscopic QED formalism [31,32], the electric field fluctuations in the presence of a surface are given as [see (A4)]

$$
\hat{\mathbf{E}}_{f}\left(\mathbf{r}^{\prime}\right)=\int d \omega \sum_{\lambda=e, m} \int d^{3} r^{\prime \prime}\left[\overline{\bar{G}}_{\lambda}\left(\mathbf{r}^{\prime}, \mathbf{r}^{\prime \prime}, \omega\right) \cdot \hat{\mathbf{f}}_{\lambda}\left(\mathbf{r}^{\prime \prime}, \omega\right)+\text { H.c. }\right],
$$

where $\overline{\bar{G}}_{\lambda}\left(\mathbf{r}_{1}, \mathbf{r}_{2}, \omega\right)$ stands for the propagator of a field excitation between points $\mathbf{r}_{1}$ and $\mathbf{r}_{2}$, as described by Eqs. (A7)(A10) [33].

Assuming that the particle has a linear, homogeneous, and isotropic polarizability $\overline{\bar{\alpha}}\left(\mathbf{r}^{\prime}, \omega\right) \equiv \alpha(\omega) \mathbb{1}$, we can write the induced polarization of the particle as $\hat{\mathbf{P}}\left(\mathbf{r}^{\prime}\right)=\mathbf{P}_{0}\left(\mathbf{r}^{\prime}, t\right)+$ $\hat{\mathbf{P}}_{f}\left(\mathbf{r}^{\prime}\right)[34]$, where

$$
\mathbf{P}_{0}\left(\mathbf{r}^{\prime}, t\right)=\frac{1}{2}\left[\alpha\left(\omega_{0}\right) \mathcal{E}_{0}\left(\mathbf{r}^{\prime}\right) e^{-i \omega_{0} t}+\text { H.c. }\right]
$$

is the polarization induced by the classical field and

$\hat{\mathbf{P}}_{f}\left(\mathbf{r}^{\prime}\right)=\int d \omega \sum_{\lambda=e, m} \int d^{3} r\left[\alpha(\omega) \overline{\bar{G}}_{\lambda}\left(\mathbf{r}^{\prime}, \mathbf{r}, \omega\right) \cdot \hat{\mathbf{f}}_{\lambda}(\mathbf{r}, \omega)+\right.$ H.c. $]$

corresponds to the polarization induced due to the fluctuations of the EM field.

We now assume that the dielectric particle is pointlike, with the c.m. position of the particle $\hat{\mathbf{r}}_{M}=\hat{\mathbb{1}} \mathbf{r}+\hat{z} \mathbf{e}_{z}$ such that $\mathbf{r}$ corresponds to the classical c.m. coordinates and $\hat{z}$ represents the quantum fluctuations of the c.m. motion along the $z$ axis about the classical trap position.

Using Eqs. (3)-(6), one can rewrite the interaction Hamiltonian Eq. (2) as

$$
\hat{H}_{\mathrm{int}}=\hat{H}_{\mathrm{Tr}}+\hat{H}_{\mathrm{CP}}+\hat{H}_{\mathrm{DCP}},
$$

where

$$
\hat{H}_{\mathrm{Tr}} \equiv-\mathbf{P}_{0}\left(\hat{\mathbf{r}}_{M}, t\right) \cdot \mathbf{E}_{0}\left(\hat{\mathbf{r}}_{M}, t\right)
$$

corresponds to the trap Hamiltonian,

$$
\hat{H}_{\mathrm{CP}} \equiv-\hat{\mathbf{P}}_{f}\left(\hat{\mathbf{r}}_{M}\right) \cdot \hat{\mathbf{E}}_{f}\left(\hat{\mathbf{r}}_{M}\right)
$$


stands for the Casimir-Polder (CP) interaction Hamiltonian, and

$$
\hat{H}_{\mathrm{DCP}}=-\left[\mathbf{E}_{0}\left(\hat{\mathbf{r}}_{M}, t\right) \cdot \hat{\mathbf{P}}_{f}\left(\hat{\mathbf{r}}_{M}\right)+\hat{\mathbf{E}}_{f}\left(\hat{\mathbf{r}}_{M}\right) \cdot \mathbf{P}_{0}\left(\hat{\mathbf{r}}_{M}, t\right)\right]
$$

is the driven Casimir-Polder (DCP) interaction. The first term in the above stands for the fluctuating dipole interacting with the classical trap field, and the second term corresponds to the classically driven dipole interacting with the EM field fluctuations at the position of the particle, as depicted by the processes (III) and (IV) in Fig. 1(b). We study each of these contributions in detail in the following.

\section{A. Classical trap}

The classical trap potential to zeroth order in the c.m. fluctuations is given as

$$
U_{\operatorname{Tr}}(\mathbf{r}) \equiv-\frac{1}{2}\left\langle\mathbf{P}_{0}(\mathbf{r}, t) \cdot \mathbf{E}_{0}(\mathbf{r}, t)\right\rangle=-\frac{1}{4} \alpha\left(\omega_{0}\right)\left|\mathcal{E}_{0}(\mathbf{r})\right|^{2},
$$

where we have taken a time average over the electric field. We note that the factor of $1 / 2$ is introduced to avoid the double sum of the energy associated with the interaction of the induced polarization and electric field [34]. We have further assumed here that the dielectric particle has negligible internal loss with a real polarizability such that $\alpha(\omega)=\alpha^{*}(\omega)$.

Expanding $\hat{H}_{\mathrm{Tr}}$ to second order in the c.m. fluctuations $\hat{z}$ around the classical equilibrium position $\mathbf{r}_{0}$, and ignoring constant energy shifts, one obtains the trap potential as $\hat{V}_{\operatorname{Tr}} \equiv$ $\frac{1}{2} M \Omega_{\mathrm{Tr}}^{2} \hat{z}^{2}$, where $\Omega_{\mathrm{Tr}}=\sqrt{\frac{\alpha\left(\omega_{0}\right) k_{0}^{2}\left|\mathcal{E}_{0}\left(\mathbf{r}_{0}\right)\right|^{2}}{2 M}}$ corresponds to the frequency of the trap due to the classical field. We have assumed here that the electric field amplitude for the standing wave formed by the classical trap field goes as $\mathcal{E}_{0}\left(\mathbf{r}_{0}\right) \sim e^{i k_{0} z}$ as a function of $z$.

\section{B. Casimir-Polder interaction}

Considering the interaction between field and polarization fluctuations to zeroth order in c.m. fluctuations $\hat{z}$, such that $\hat{H}_{\mathrm{CP}}^{(0)} \equiv-\hat{\mathbf{P}}_{f}(\mathbf{r}) \cdot \hat{\mathbf{E}}_{f}(\mathbf{r})$, one can obtain the Casimir-Polder potential as $U_{\mathrm{CP}}(\mathbf{r}) \equiv \frac{1}{2} \operatorname{Tr}_{B}\left[\hat{\rho}_{F} \hat{H}_{\mathrm{CP}}^{(0)}\right]$. This can be evaluated in first-order perturbation theory as [32]

$$
\begin{aligned}
U_{\mathrm{CP}}(\mathbf{r})= & \frac{\hbar \mu_{0}}{2 \pi} \int_{0}^{\infty} d \xi \xi^{2} \alpha(i \xi) \operatorname{Tr}\left[\overline{\bar{G}}_{\mathrm{sc}}(\mathbf{r}, \mathbf{r}, i \xi)\right] \\
& -\frac{\hbar \mu_{0}}{\pi} \int d \omega \omega^{2} n_{\mathrm{th}}(\omega) \alpha(\omega) \operatorname{Tr}\left[\operatorname{Im} \overline{\bar{G}}_{\mathrm{sc}}(\mathbf{r}, \mathbf{r}, \omega)\right],
\end{aligned}
$$

where we have assumed that the field density matrix $\hat{\rho}_{F}$ corresponds to a thermal state with temperature $T$ and $n_{\text {th }}(\omega)=$ $\left\langle\hat{\mathbf{f}}_{\lambda}^{\dagger}(\mathbf{r}, \omega) \cdot \hat{\mathbf{f}}_{\lambda}(\mathbf{r}, \omega)\right\rangle=\frac{1}{e^{\hbar \omega /\left(k_{B} T\right)}-1}$ is the average number of thermal photons in the mode $\omega$. All surface properties enter into consideration through the scattering Green's tensor $\overline{\bar{G}}_{\text {sc }}(\mathbf{r}, \mathbf{r}, i \xi)$ [see Eq. (A10)] corresponding to the propagation of a virtual photon from the position (r) of the particle to the surface and back. Given that imaginary frequencies are associated with virtual interactions, the above potential can be physically understood as coming from the interaction between the fluctuations of the dipole and those of the vacuum EM field, summed over all frequencies of virtual photons exchanged between the particle and the surface. The second term corresponds to the scattering and reabsorption of thermal fluctuations of the EM field by the particle off the surface.

\section{Drive-induced Casimir-Polder interaction}

The linearized part of the interaction Hamiltonian with respect to the classical field gives a drive-induced contribution to zeroth order in the c.m. fluctuations given by

$$
\hat{H}_{\mathrm{DCP}}^{(0)} \equiv-\left[\mathbf{E}_{0}(\mathbf{r}, t) \cdot \hat{\mathbf{P}}_{f}(\mathbf{r})+\hat{\mathbf{E}}_{f}(\mathbf{r}) \cdot \mathbf{P}_{0}(\mathbf{r}, t)\right] .
$$

One can derive a corresponding drive-induced Casimir-Polder potential in second-order perturbation theory as [35] (see Appendix $\mathrm{C}$ for details)

$$
\begin{aligned}
U_{\mathrm{DCP}}(\mathbf{r})= & -\frac{\mu_{0} \omega_{0}^{2}\left[\alpha\left(\omega_{0}\right)^{2}\right]}{2}\left[2 n_{\mathrm{th}}\left(\omega_{0}+1\right)\right] \\
& \times\left[\mathcal{E}_{0}(\mathbf{r}) \cdot \operatorname{Re} \overline{\bar{G}}_{\mathrm{sc}}\left(\mathbf{r}, \mathbf{r}, \omega_{0}\right) \cdot \mathcal{E}_{0}^{*}(\mathbf{r})\right] .
\end{aligned}
$$

The above shift is analogous to the resonant Casimir-Polder shift for the excited state of a two-level atom [32,35,36]. This can be understood as coming from a process wherein a classically induced dipole scatters a photon off of the surface and reabsorbs it.

\section{Total potential}

The total potential for the classical coordinate of the particle can be written as the sum of Eqs. (11), (12), and (14) as $U_{\mathrm{Tot}}(\mathbf{r})=U_{\mathrm{Tr}}(\mathbf{r})+U_{\mathrm{CP}}(\mathbf{r})+U_{\mathrm{DCP}}(\mathbf{r})$, which yields the classical equilibrium position $\mathbf{r}_{0}$ of the particle such that $\left.\partial_{z} U_{\text {Tot }}(\mathbf{r})\right|_{\mathbf{r}_{0}}=0$.

Expanding the CP and DCP Hamiltonians to second order in $\hat{z}$ around $\mathbf{r}_{0}$ would lead to additional corrections to the trap frequency. Assuming that the potential associated with the CP and DCP contributions are $\hat{V}_{(\mathrm{D}) \mathrm{CP}} \equiv \frac{1}{2} M \Omega_{(\mathrm{D}) \mathrm{CP}}^{2} \hat{z}^{2}$, the total free Hamiltonian for the particle is the sum of its kinetic energy term and the harmonic trap potential given by

$$
\hat{H}_{M}=\frac{\hat{p}_{z}^{2}}{2 M}+\frac{1}{2} M \Omega^{2} \hat{z}^{2},
$$

where the total trap frequency is defined as $\Omega \equiv$ $\sqrt{\Omega_{\mathrm{Tr}}^{2}+\Omega_{\mathrm{CP}}^{2}+\Omega_{\mathrm{DCP}}^{2}}$.

\section{QBM FOR THE PARTICLE IN THE PRESENCE OF A SURFACE}

We now study the open system dynamics of the quantized c.m. motion of the particle as the system of interest, interacting with the fluctuations of the EM field as the bath. It can be seen that in the presence of an external trapping field, to the lowest order in field fluctuations, the coupling between the quantized c.m. motion and the EM field fluctuations arises due to the drive-induced Casimir-Polder Hamiltonian $\hat{H}_{\mathrm{DCP}}{ }^{1}$ Expanding $\hat{H}_{\mathrm{DCP}}$ to first order in the c.m. motion fluctuations,

\footnotetext{
${ }^{1}$ The c.m. also couples to the vacuum fluctuations of the field via $\hat{H}_{\text {CP. }}$ We remark that the Brownian motion of the classical c.m. due to vacuum fluctuations has been previously analyzed in Ref. [37].
} 
we obtain an interaction Hamiltonian between the quantized c.m. motion and the fluctuations of the field as

$$
\hat{H}_{M F}(t) \approx \hat{z} \hat{\mathcal{B}}(t)
$$

where $\hat{\mathcal{B}}(t)$ is the bath operator defined as

$$
\begin{aligned}
\hat{\mathcal{B}}(t)= & -\left[\mathbf{P}_{0}\left(\mathbf{r}_{0}, t\right) \cdot \frac{\partial}{\partial z} \hat{\mathbf{E}}_{f}\left(\mathbf{r}_{0}\right)+\frac{\partial}{\partial z} \hat{\mathbf{P}}_{f}\left(\mathbf{r}_{0}\right) \cdot \mathbf{E}_{0}\left(\mathbf{r}_{0}, t\right)\right. \\
& \left.+\frac{\partial}{\partial z} \mathbf{P}_{0}\left(\mathbf{r}_{0}, t\right) \cdot \hat{\mathbf{E}}_{f}\left(\mathbf{r}_{0}\right)+\hat{\mathbf{P}}_{f}\left(\mathbf{r}_{0}\right) \cdot \frac{\partial}{\partial z} \mathbf{E}_{0}\left(\mathbf{r}_{0}, t\right)\right] .
\end{aligned}
$$

We remark that $\hat{\mathcal{B}}(t)$ physically corresponds to the force on the c.m.. While the last two terms in the above with $\partial_{z} \mathbf{E}_{0}(\mathbf{r})$ and $\partial_{z} \mathbf{P}_{0}(\mathbf{r})$ vanish at the field intensity maxima, the equilibrium position $\mathbf{r}_{0}$ is shifted from that point due to the presence of the $\mathrm{CP}$ and DCP potentials.

Moving to a rotating frame of reference with respect to the total free Hamiltonian, we can write the interaction Hamiltonian [Eq. (16)] in the interaction picture as $\tilde{H}_{M F}(t) \equiv$ $e^{-i\left(\hat{H}_{M}+\hat{H}_{F}\right) t} \hat{H}_{M F}(t) e^{i\left(\hat{H}_{M}+\hat{H}_{F}\right) t}$. In the interaction picture we can thus describe the dynamics of the c.m. in terms of a BornMarkov master equation as [27]

$\frac{d \hat{\rho}_{M}}{d t}=-\frac{1}{\hbar^{2}} \operatorname{Tr}_{B} \int_{0}^{\infty} d \tau\left[\tilde{H}_{M F}(t),\left[\tilde{H}_{M F}(t-\tau), \hat{\rho}_{M}(t) \otimes \hat{\rho}_{F}\right]\right]$

where $\hat{\rho}_{M}$ refers to the density matrix for the quantized c.m. motion of the particle. Performing a trace over the bath in the above, one obtains the following QBM master equation,

$$
\begin{aligned}
\frac{d \hat{\rho}_{M}}{d t}= & \frac{1}{2 \hbar^{2}} \int_{0}^{\infty} d \tau\left[i \mathcal{D}(\tau) \cos (\Omega \tau)\left[\hat{z},\left\{\hat{z}, \hat{\rho}_{M}\right\}\right]\right. \\
& -i \mathcal{D}(\tau) \frac{\sin (\Omega \tau)}{M \Omega}\left[\hat{z},\left\{\hat{p}_{z}, \hat{\rho}_{M}\right\}\right]-\mathcal{N}(\tau) \cos (\Omega \tau) \\
& \left.\times\left[\hat{z},\left[\hat{z}, \hat{\rho}_{M}\right]\right]+\mathcal{N}(\tau) \frac{\sin (\Omega \tau)}{M \Omega}\left[\hat{z},\left[\hat{p}_{z}, \hat{\rho}_{M}\right]\right]\right],
\end{aligned}
$$

where the first term corresponds to trap frequency renormalization, the second term corresponds to dissipation or friction, the third term represents decoherence in the position basis, and the last term corresponds to momentum diffusion. The dissipation and noise kernels in the above master equation are given as (see Appendix D for details of the derivation)

$$
\begin{gathered}
\mathcal{D}(\tau) \equiv i\langle[\tilde{\mathcal{B}}(t), \tilde{\mathcal{B}}(t-\tau)]\rangle \\
=2 \hbar \int_{0}^{\infty} d \omega J\left(\omega, \mathbf{r}_{0}\right) \sin (\omega \tau) \cos \left(\omega_{0} \tau\right), \\
\mathcal{N}(\tau) \equiv\langle\{\tilde{\mathcal{B}}(t), \tilde{\mathcal{B}}(t-\tau)\}\rangle \\
=2 \hbar \int_{0}^{\infty} d \omega J\left(\omega, \mathbf{r}_{0}\right) \cos (\omega \tau) \cos \left(\omega_{0} \tau\right) \operatorname{coth}\left(\frac{\hbar \omega}{2 k_{B} T}\right),
\end{gathered}
$$

where we have defined the bath operator in the interaction picture as $\tilde{\mathcal{B}}(t) \equiv e^{-i\left(\hat{H}_{M}+\hat{H}_{F}\right) t} \hat{\mathcal{B}}(t) e^{i\left(\hat{H}_{M}+\hat{H}_{F}\right) t}$. The kernels $\mathcal{D}(\tau)$ and $\mathcal{N}(\tau)$ correspond to the standard QBM dissipation and noise kernels, respectively. Here, $J\left(\omega, \mathbf{r}_{0}\right) \equiv J_{\text {free }}\left(\omega, \mathbf{r}_{0}\right)+$ $J_{\text {sc }}\left(\omega, \mathbf{r}_{0}\right)$ is the effective spectral density of the EM field in the presence of the surface, with the free-space and scattering contributions given by

$$
J_{\text {free }, \mathrm{sc}}\left(\omega, \mathbf{r}_{0}\right)=\frac{\omega^{2}}{2 \pi \epsilon_{0} c^{2}}\left[\alpha\left(\omega_{0}\right)+\alpha(\omega)\right]^{2} g_{\text {free }, \mathrm{sc}}\left(\omega, \mathbf{r}_{0}\right),
$$

where we have defined

$$
\begin{aligned}
g_{\text {free }, \mathrm{sc}}\left(\omega, \mathbf{r}_{0}\right) \equiv & \mathcal{E}_{0}\left(\mathbf{r}_{0}\right) \cdot\left\{\partial_{z} \operatorname{Im} \overline{\bar{G}}_{\text {free }, \mathrm{sc}}\left(\mathbf{r}_{0}, \mathbf{r}_{0}, \omega\right) \partial_{z}\right\} \cdot \mathcal{E}_{0}^{*}\left(\mathbf{r}_{0}\right) \\
& +\partial_{z} \mathcal{E}_{0}\left(\mathbf{r}_{0}\right) \cdot \operatorname{Im} \overline{\bar{G}}_{\text {free }, \mathrm{sc}}\left(\mathbf{r}_{0}, \mathbf{r}_{0}, \omega\right) \cdot \partial_{z} \mathcal{E}_{0}^{*}\left(\mathbf{r}_{0}\right) \\
& +\mathcal{E}_{0}\left(\mathbf{r}_{0}\right) \cdot\left\{\partial_{z} \operatorname{Im} \overline{\bar{G}}_{\text {free }, \mathrm{sc}}\left(\mathbf{r}_{0}, \mathbf{r}_{0}, \omega\right)\right\} \cdot \partial_{z} \mathcal{E}_{0}^{*}\left(\mathbf{r}_{0}\right) \\
& +\partial_{z} \mathcal{E}_{0}\left(\mathbf{r}_{0}\right) \cdot\left\{\operatorname{Im} \overline{\bar{G}}_{\text {free }, \mathrm{sc}}\left(\mathbf{r}_{0}, \mathbf{r}_{0}, \omega\right) \partial_{z}\right\} \cdot \mathcal{E}_{0}^{*}\left(\mathbf{r}_{0}\right)
\end{aligned}
$$

To physically interpret the spectral density obtained above, we note the following features from Eq. (22):

(1) The spectral density scales as the square of the induced dipole of the dielectric particle. The part of total spectral density that depends on $\left[\alpha\left(\omega_{0}\right)\right]^{2}$ arises due to the classically induced dipole, corresponding to the emission and reabsorption of a photon by the classical dipole. The part of the spectral density that depends on $[\alpha(\omega)]^{2}$ arises from the interactions of the fluctuating dipole with its image via the classical trap field. Terms that go as $\sim \alpha\left(\omega_{0}\right) \alpha(\omega)$ can be understood as coming from processes where a classical dipole scatters a photon, inducing a fluctuating dipole in the medium, which in turn interacts with the classical dipole via the trap field. This can be seen from the derivation of the dissipation and noise kernels in Appendix D.

(2) Given that the imaginary part of the surface scattering Green's tensor Im $\overline{\bar{G}}_{\text {sc }}$ corresponds to the surface loss, the density of modes increases near a lossy surface. This indicates that a lossy surface with a large number of fluctuating degrees of freedom leads to a larger dissipation and decoherence for the quantized c.m. dynamics, as a result of the fluctuationdissipation theorem. Previously it has also been shown that surface loss leads to additional dissipation and decoherence for the internal degrees of the particle [18-24].

(3) In addition to the surface-induced modifications, there is also dissipation and decoherence due to the interaction of the particle with the free-space EM field modes, as given by the free-space Green's tensor contribution. This can be understood as arising from scattering of the classical drive photons by the particle into free-space modes.

We can now define the dissipation and decoherence coefficients as

$$
\begin{aligned}
\Gamma \equiv & \frac{1}{2 \hbar M \Omega} \int_{0}^{\infty} d \tau \mathcal{D}(\tau) \sin (\Omega \tau) \\
= & \frac{\pi}{4 M \Omega}\left[J\left(\omega_{0}+\Omega, \mathbf{r}_{0}\right)-J\left(\omega_{0}-\Omega, \mathbf{r}_{0}\right)\right] \\
\Lambda \equiv & \frac{1}{2 \hbar^{2}} \int_{0}^{\infty} d \tau \mathcal{N}(\tau) \cos (\Omega \tau) \\
= & \frac{\pi}{4 \hbar}\left[J\left(\omega_{0}+\Omega, \mathbf{r}_{0}\right) \operatorname{coth}\left(\frac{\hbar\left(\omega_{0}+\Omega\right)}{k_{B} T}\right)\right. \\
& \left.\quad+J\left(\omega_{0}-\Omega, \mathbf{r}_{0}\right) \operatorname{coth}\left(\frac{\hbar\left(\omega_{0}-\Omega\right)}{k_{B} T}\right)\right]
\end{aligned}
$$


This shows that the dissipation and decoherence depend only on the effective spectral density evaluated at the mechanical sideband frequencies of the driving field. It can be seen that the above expressions are analogous to those for optomechanical damping and radiation pressure-induced noise [38].

This allows us to simplify the master equation Eq. (19) as follows,

$$
\frac{d \hat{\rho}_{M}}{d t} \approx-\frac{i}{\hbar}\left[H_{M}^{\prime}, \hat{\rho}_{M}\right]-\frac{i \Gamma}{\hbar}\left[\hat{z},\left\{\hat{p}_{z}, \hat{\rho}_{M}\right\}\right]-\Lambda\left[\hat{z},\left[\hat{z}, \hat{\rho}_{M}\right]\right],
$$

where we have defined the renormalized free Hamiltonian for the center of mass as $H_{M}^{\prime}$ which includes the frequency renormalization due to the first term in Eq. (19), and ignored the momentum diffusion term [27].

\section{DECOHERENCE AND QUANTUM FRICTION FOR A DIELECTRIC NANOSPHERE}

As a concrete example, we now evaluate the decoherence and dissipation for a dielectric nanosphere near a planar half space. The parameter values corresponding to the particle and surface, and simplifying assumptions are given as follows.

\section{A. Parameter values and assumptions}

(1) We consider a dielectric nanosphere made of silica with a radius $R=72 \mathrm{~nm}$, and density $\rho \approx 2000 \mathrm{~kg} / \mathrm{m}^{3}$ [16].

(2) The polarizability of a dielectric nanosphere is given as [34]

$$
\overline{\bar{\alpha}}(\omega)=3 \epsilon_{0} \mathcal{V}\left[\frac{\epsilon_{P}(\omega)-1}{\epsilon_{P}(\omega)+2}\right] \mathbb{1},
$$

where $\mathcal{V}=\frac{4}{3} \pi R^{3}$ is the volume of the nanosphere, and $\epsilon_{P}(\omega)$ is the dielectric permittivity of the dielectric particle described by the Drude-Lorentz model

$$
\epsilon_{P}(\omega)=1+\frac{\omega_{p 1}^{2}}{\omega_{T 1}^{2}-\omega^{2}-i \gamma_{1} \omega}+\frac{\omega_{p 2}^{2}}{\omega_{T 2}^{2}-\omega^{2}-i \gamma_{2} \omega} .
$$

We use the parameters corresponding to fused silica as $\omega_{p 1}=1.75 \times 10^{14} \mathrm{~Hz}, \gamma_{1}=4.28 \times 10^{13} \mathrm{~Hz}, \omega_{T 1}=1.32 \times$ $10^{14} \mathrm{~Hz}, \omega_{p 2}=2.96 \times 10^{16} \mathrm{~Hz}, \gamma_{2}=8.09 \times 10^{15} \mathrm{~Hz}, \omega_{T 2}=$ $2.72 \times 10^{16} \mathrm{~Hz}$ [39]. In the present calculations we will ignore the damping and consider only the real part of the total polarizability.

(3) We assume the trap field to be polarized along the $x$ axis, with a wavelength of $\lambda_{0} \approx 1064 \mu \mathrm{m}$ and intensity $I=$ $\frac{1}{2} \epsilon_{0} \mathcal{E}_{0}^{2} c \approx 10^{-11} \mathrm{~W} \mathrm{~m}^{-2}$, as used in Ref. [16].

(4) We assume that the particle is trapped in a harmonic potential along the $z$ axis, with a trap frequency $\Omega \approx 3 \mathrm{MHz}$.

(5) Considering that the classical drive frequency is much larger than that for the mechanical trap $\omega_{0} \gg \Omega$, and $\hbar \omega_{0} \gg$ $k_{B} T$, one can simplify Eqs. (24) and (25) to obtain the following simple expressions for the dissipation and noise,

$$
\begin{gathered}
\Gamma \approx \frac{\pi}{2 M} J^{\prime}\left(\omega_{0}, \mathbf{r}_{0}\right), \\
\Lambda \approx \frac{\pi}{2 \hbar} J\left(\omega_{0}, \mathbf{r}_{0}\right) .
\end{gathered}
$$

(6) For the purpose of estimation we consider in the following that the equilibrium position is roughly given by the classical trap field intensity maximum, such that we can approximate the spectral density in Eq. (22) as

$J_{\text {free, }, \mathrm{sc}}\left(\omega, \mathbf{r}_{0}\right) \approx \frac{\omega^{2}}{2 \pi \epsilon_{0} c^{2}}\left[\alpha\left(\omega_{0}\right)+\alpha(\omega)\right]^{2}\left|\mathcal{E}_{0}\left(\mathbf{r}_{0}\right)\right|^{2} \mathcal{G}_{\text {free, }, \mathrm{sc}}\left(\omega, \mathbf{r}_{0}\right)$,

where we have assumed that $\partial_{z} \mathcal{E}_{0}\left(\mathbf{r}_{0}\right) \approx \mathbf{0}$ and defined the free-space and scattering recoil Green's tensor as

$$
\mathcal{G}_{\text {sc,free }}\left(\omega, \mathbf{r}_{0}\right) \equiv \mathbf{e}_{x} \cdot \partial_{z} \operatorname{Im} \overline{\bar{G}}_{\text {free, sc }}\left(\mathbf{r}_{0}, \mathbf{r}_{0}, \omega\right) \partial_{z} \cdot \mathbf{e}_{x},
$$

where we have assumed the trap field to be polarized along the $x$ axis.

\section{B. Surface properties}

We calculate the influence of the free-space and surface scattered EM field given by the the imaginary part of the Green's tensor as follows.

\section{Free space}

Observing that the free-space recoil Green's tensor is $\mathcal{G}_{\text {free }}\left(\omega, \mathbf{r}_{0}\right)=\frac{\omega^{3}}{15 \pi c^{3}}$ [see Eq. (B8)], and using Eq. (31), the contribution to the spectral density due to the free-space EM field modes is given by

$$
J_{\text {free }}(\omega)=\frac{2 \hbar \omega^{2} \gamma_{0}(\omega)}{5 \pi c^{2}},
$$

where we have defined

$$
\gamma_{0}(\omega) \equiv \frac{\left[\alpha\left(\omega_{0}\right)+\alpha(\omega)\right]^{2}\left|\mathcal{E}_{0}\right|^{2} \omega^{3}}{12 \pi \epsilon_{0} \hbar c^{3}},
$$

analogous to the dissipation rate of a dipole of strength $d \equiv$ $\left[\frac{\alpha\left(\omega_{0}\right)+\alpha(\omega)}{2}\right]\left|\mathcal{E}_{0}\right|$ interacting with the vacuum EM field. It can be seen from Eq. (30) that this yields a decoherence rate of

$$
\Lambda_{\text {free }}=\frac{\omega_{0}^{2} \gamma_{0}\left(\omega_{0}\right)}{5 c^{2}}
$$

which corresponds to the position decoherence of the particle arising from the scattering of drive photons into free-space modes.

\section{Perfect conductor}

For a perfectly conducting planar surface (with the Fresnel coefficients $r_{p}=1$ and $r_{s}=-1$ ) the scattering part of the recoil Green's tensor is given as [see Eq. (B6)]

$$
\begin{aligned}
\mathcal{G}_{\mathrm{sc}, \mathrm{pc}}\left(\omega, \mathbf{r}_{0}\right)= & \frac{\omega^{3}}{32 \pi \tilde{z}^{5} c^{3}}\left[\left(\tilde{z}^{2}-1\right)\{6 \tilde{z} \cos (2 \tilde{z})\right. \\
& \left.\left.+\left(4 \tilde{z}^{2}-3\right) \sin (2 \tilde{z})\right\}\right],
\end{aligned}
$$

where $\tilde{z} \equiv k_{0} z$ is the dimensionless distance of the particle from the surface. In the near-field limit $\tilde{z} \ll 1$, we find that $\mathcal{G}_{\mathrm{sc}, \mathrm{pc}}^{\mathrm{NR}}\left(\omega, \mathbf{r}_{0}\right) \approx \frac{\omega^{3}}{15 \pi c^{3}}$, where NR stands for the nonretarded regime. This yields the density of modes in the subwavelength 

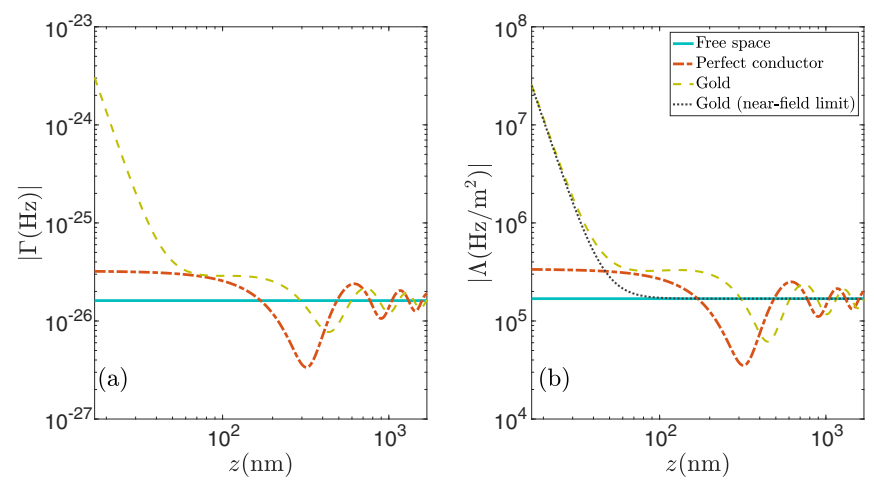

FIG. 2. (a) Dissipation and (b) decoherence of a silica nanosphere near perfect conductor and gold surfaces. The free-space dissipation and decoherence is depicted by the blue solid line in both the plots. The dotted line in (b) denotes the near-field asymptotic expression for decoherence of the particle near a metal surface as given by Eq. (42). The gold surface is described by the Drude model with a plasma frequency $\omega_{p} \approx 1.37 \times 10^{16} \mathrm{~Hz}(9 \mathrm{eV})$, and loss parameter $\gamma \approx 5.31 \times 10^{13} \mathrm{~Hz}(35 \mathrm{meV})$ [40].

limit as

$$
J_{\mathrm{pc}}^{\mathrm{NR}}\left(\omega, \mathbf{r}_{0}\right) \approx J_{\text {free }}\left(\omega, \mathbf{r}_{0}\right)+J_{\mathrm{sc}}^{\mathrm{NR}}\left(\omega, \mathbf{r}_{0}\right) \approx \frac{4 \hbar \omega^{2} \gamma_{0}(\omega)}{5 \pi c^{2}}
$$

Comparing with Eq. (33), we see that the density of modes is twice that of the free space, which can be understood as a sum of the field radiated by the dipole and its image.

The corresponding localization parameter is given as

$$
\Lambda_{\mathrm{pc}}^{\mathrm{NR}} \approx \frac{2 \omega_{0}^{2} \gamma_{0}\left(\omega_{0}\right)}{5 c^{2}}
$$

We note that in the absence of surface losses the decoherence of the particle in the near-field regime is independent of its distance from the surface, as can be seen from Fig. 2(b).

\section{Metal}

For a metal surface we assume the permittivity function to be given by the Drude model

$$
\epsilon_{S}(\omega)=1-\frac{\omega_{p}^{2}}{\omega^{2}+i \omega \gamma}
$$

The scattering recoil Green's tensor is given as [see Eq. (B6)]

$$
\begin{aligned}
\mathcal{G}_{\mathrm{sc}, \mathrm{met}}\left(\omega, \mathbf{r}_{0}\right)= & \frac{1}{8 \pi} \operatorname{Im}\left[\int_{0}^{\infty} d k_{\|} k_{\|} \kappa_{\perp} e^{-2 \kappa_{\perp} z}\right. \\
& \left.\times\left\{r_{p}\left(\kappa_{\perp}, k_{0}\right) \frac{\kappa_{\perp}^{2}}{k_{0}^{2}}+r_{s}\left(\kappa_{\perp}, k_{0}\right)\right\}\right],
\end{aligned}
$$

where the Fresnel coefficients are as given by Eq. (B2).

In the near-field limit the scattering recoil Green's tensor can be simplified to $\mathcal{G}_{\mathrm{sc}, \text { met }}^{\mathrm{NR}}\left(\omega, \mathbf{r}_{0}\right) \approx \frac{3 \omega^{3}}{32 \pi \tilde{z}^{5} c^{3}} \operatorname{Im}\left[\frac{\epsilon_{S}(\omega)-1}{\epsilon_{S}(\omega)+1}\right]$, yielding a density of modes near a metal surface as

$$
J_{\mathrm{met}}^{\mathrm{NR}}\left(\omega, \mathbf{r}_{0}\right) \approx \frac{9 \hbar \omega^{2}}{16 \pi c^{2} \tilde{z}^{5}} \operatorname{Im}\left[\frac{\epsilon_{S}(\omega)-1}{\epsilon_{S}(\omega)+1}\right] \gamma_{0}(\omega) .
$$

One can thus write the decoherence of the particle near a metal half space as

$$
\Lambda_{\mathrm{met}}^{\mathrm{NR}} \approx \frac{9 k_{0}^{2}}{32 \tilde{z}^{5}} \operatorname{Im}\left[\frac{\epsilon_{S}\left(\omega_{0}\right)-1}{\epsilon_{S}\left(\omega_{0}\right)+1}\right] \gamma_{0}\left(\omega_{0}\right) \approx \frac{3}{4 z^{2}} \gamma_{\mathrm{sc}}\left(\mathbf{r}_{0}\right),
$$

where $\gamma_{\mathrm{sc}}\left(\mathbf{r}_{0}\right) \approx \frac{3}{8 \tilde{z}^{3}} \operatorname{Im}\left[\frac{\epsilon_{S}\left(\omega_{0}\right)-1}{\epsilon_{S}\left(\omega_{0}\right)+1}\right] \gamma_{0}\left(\omega_{0}\right)$ is surface-modified photon scattering rate [see Eq. (C16)]. As seen from Fig. 2(b), the decoherence of the particle's c.m. in the near-field regime is well approximated by the above expression.

\section{Correspondence to collisional model of decoherence}

We note that the deocherence term in the master equation Eq. (26) is of the position localization decoherence (PLD) form [30]. To understand this, we note that a similar form of a decoherence term can also be obtained from a collisional model of decoherence, wherein the system in consideration is bombarded by individual scatterers from the environment. As each scattering bath particle interacts with the system via a local interaction and gets correlated, it acquires some information about the system's position as a result. Thus, upon tracing out the bath, the system exhibits decoherence in the position basis. Particularly in the limit where the scatterer has a much longer de Broglie wavelength compared to the coherence length scale of the system, one obtains a decoherence term as in Eq. (26) [29,30]. This correspondence in the decoherence dynamics from two different models suggests that the decoherence of a particle near a surface arises due to scattering of virtual photons off of the surface.

We note that the decoherence rate due to scattering of photons in free space goes as $\Lambda_{\text {free }} \sim k_{\text {eff }}^{2} \gamma_{\text {eff }}$ [see Eq. (35)], where $k_{\text {eff }}$ refers to an effective wave vector for the scattered photon and $\gamma_{\text {eff }}$ is the rate of scattering. Considering that $\Lambda_{\text {met }}^{\mathrm{NR}} \sim \gamma_{\mathrm{sc}}\left(\mathbf{r}_{0}\right) / z^{2}$, we deduce from Eq. (42) that the virtual photons inducing decoherence have an effective de Broglie wavelength $\sim k_{\text {eff }}^{-1} \sim z$ that scales as the distance of the particle from the surface. We remark that a similar effective de Broglie wavelength was previously also derived in Ref. [36] in the context of recoil heating of a driven atom near a surface.

\section{DISCUSSION}

To summarize, we have derived a quantum Brownian motion master equation for the quantized center-of-mass motion of a dielectric particle trapped near a surface. Considering the particle to be trapped with an external classical field, we find that there are three different contributions to the total potential seen by the particle - a classical trap potential, Casimir-Polder potential, and driven CP potential, as illustrated in Fig. 1(b). Taken together, these potentials lead the particle to be trapped close to the first intensity maxima of the standing-wave potential formed by the classical field (see Sec. II D). The interaction between the quantized c.m. motion and the fluctuations of the EM field to the lowest order is described by a linear expansion of the driven CP potential [Eq. (10)] about the equilibrium position. Tracing out the EM field fluctuations, we arrive at a second-order Born-Markov master equation describing the dissipative dynamics of the 
particle's c.m. as given by Eq. (19). The resulting dynamics is governed by a quantum Brownian motion master equation, with an effective spectral density that is determined by the polarizability of the particle, properties of the surface, and the strength of the external trapping field [Eq. (22)]. The dissipation and decoherence that arise as a result can be understood as coming from the classically induced dipole scattering field fluctuations, and the fluctuating dipole scattering the drive photons. We then estimate the decoherence and dissipation for a dielectric nanosphere near different surfaces, and find that the quantized c.m. decoherence and dissipation increase in the presence of a lossy medium (Fig. 2). We further illustrate a correspondence between the resulting decoherence and that from the collisional model in the long-wavelength limit $[29,30]$.

Comparing the resulting decoherence due to surface fluctuations with that arising from other sources as a benchmark, we observe that surface-induced decoherence can potentially pose a fundamental limit for preparing a dielectric particle in macroscopic c.m. quantum states. It can be seen from Appendix $\mathrm{E}$ that the decoherence due to background gas scattering and blackbody radiation can be reduced significantly by going to lower pressures and temperatures, respectively. In the present analysis we have derived the spectral density that governs the surface modifications to fluctuation phenomena for the quantized c.m. of a particle. This could allow one to systematically modify the surface properties and drive strength in order to mitigate the surfaceinduced dissipation and decoherence. As quantum optical systems are being increasingly miniaturized, and mesoscopic quantum components being regularly interfaced with surfaces and waveguides at nanoscales, our results provide insights into tailoring fluctuation phenomena in these regimes [41-44].

\section{ACKNOWLEDGMENTS}

We are specially grateful to P. W. Milonni for insightful discussions and thoughtful feedback on the manuscript. We thank M. Aspelmeyer, U. Delić, E. A. Goldschmidt, W. D. Hodson, S. Hong, B.-L. Hu, L. Magrini, P. Meystre, and O. Romero-Isart for helpful discussions. K.S. would like to thank the Aspelmeyer group for graciously hosting her visit at the University of Vienna where part of this work was carried out. Y.S. acknowledges support from the Los Alamos National Laboratory ASC Beyond Moore's Law project and the LDRD program. The authors enjoyed the hospitality of IHoP (International House of Physicists) during the foundational stages of this work.

\section{APPENDIX A: MEDIUM-ASSISTED EM FIELD}

Using the macroscopic QED formalism [31,32], the Hamiltonian for the vacuum EM field in the presence of the surface can be written as

$$
H_{F}=\sum_{\lambda=e, m} \int d^{3} r \int d \omega \hbar \omega \hat{\mathbf{f}}_{\lambda}^{\dagger}(\mathbf{r}, \omega) \cdot \hat{\mathbf{f}}_{\lambda}(\mathbf{r}, \omega),
$$

with $\hat{\mathbf{f}}_{\lambda}^{\dagger}(\mathbf{r}, \omega)$ and $\hat{\mathbf{f}}_{\lambda}(\mathbf{r}, \omega)$ as the bosonic creation and annihilation operators, respectively, that take into account the presence of the media. Physically, these can be understood as the ladder operators corresponding to the noise polarization $(\lambda=e)$ and magnetization $(\lambda=m)$ in the medium-assisted EM field, at frequency $\omega$, created or annihilated at position r. The medium-assisted bosonic operators obey the canonical commutation relations

$$
\begin{gathered}
{\left[\hat{\mathbf{f}}_{\lambda}(\mathbf{r}, \omega), \hat{\mathbf{f}}_{\lambda^{\prime}}\left(\mathbf{r}^{\prime}, \omega^{\prime}\right)\right]=\left[\hat{\mathbf{f}}_{\lambda}^{\dagger}(\mathbf{r}, \omega), \hat{\mathbf{f}}_{\lambda^{\prime}}^{\dagger}\left(\mathbf{r}^{\prime}, \omega^{\prime}\right)\right]=\mathbf{0},} \\
{\left[\hat{\mathbf{f}}_{\lambda}(\mathbf{r}, \omega), \hat{\mathbf{f}}_{\lambda^{\prime}}^{\dagger}\left(\mathbf{r}^{\prime}, \omega^{\prime}\right)\right]=\delta_{\lambda \lambda^{\prime}} \delta\left(\mathbf{r}-\mathbf{r}^{\prime}\right) \delta\left(\omega-\omega^{\prime}\right) .}
\end{gathered}
$$

The electric and magnetic field operators evaluated at the position of the particle are given as

$$
\begin{gathered}
\hat{\mathbf{E}}_{f}\left(\mathbf{r}_{0}\right)=\sum_{\lambda=e, m} \int d^{3} r \int d \omega\left[\overline{\bar{G}}_{\lambda}\left(\mathbf{r}_{0}, \mathbf{r}, \omega\right) \cdot \hat{\mathbf{f}}_{\lambda}(\mathbf{r}, \omega)+\text { H.c. }\right], \\
\hat{\mathbf{B}}_{f}\left(\mathbf{r}_{0}\right)=\sum_{\lambda=e, m} \int d^{3} r \int d \omega\left[\left(-\frac{i}{\omega}\right)\left[\nabla \times \overline{\bar{G}}_{\lambda}\left(\mathbf{r}_{0}, \mathbf{r}, \omega\right)\right]\right. \\
\left.\cdot \hat{\mathbf{f}}_{\lambda}(\mathbf{r}, \omega)+\text { H.c. }\right],
\end{gathered}
$$

respectively, where

$$
\left[\nabla \times \overline{\bar{G}}_{\lambda}\left(\mathbf{r}, \mathbf{r}^{\prime} \omega\right)\right]_{i l}=\epsilon_{i j k} \partial_{r_{j}}\left[\overline{\bar{G}}_{\lambda}\left(\mathbf{r}, \mathbf{r}^{\prime}, \omega\right)\right]_{k l} .
$$

The coefficients $\overline{\bar{G}}_{\lambda}\left(\mathbf{r}_{1}, \mathbf{r}_{2}, \omega\right)$ are defined as

$$
\begin{gathered}
\overline{\bar{G}}_{e}\left(\mathbf{r}, \mathbf{r}^{\prime}, \omega\right)=i \frac{\omega^{2}}{c^{2}} \sqrt{\frac{\hbar}{\pi \epsilon_{0}} \operatorname{Im}\left[\epsilon\left(\mathbf{r}^{\prime}, \omega\right)\right]} \overline{\bar{G}}\left(\mathbf{r}, \mathbf{r}^{\prime}, \omega\right), \\
\overline{\bar{G}}_{m}\left(\mathbf{r}, \mathbf{r}^{\prime}, \omega\right)=\frac{i \omega}{c} \sqrt{\frac{\hbar}{\pi \epsilon_{0}} \frac{\operatorname{Im}\left[\mu\left(\mathbf{r}^{\prime}, \omega\right)\right]}{\left|\mu\left(\mathbf{r}^{\prime}, \omega\right)\right|^{2}}}\left[\nabla^{\prime} \times \overline{\bar{G}}\left(\mathbf{r}^{\prime}, \mathbf{r}, \omega\right)\right]^{T},
\end{gathered}
$$

with $\epsilon(\mathbf{r}, \omega)$ and $\mu(\mathbf{r}, \omega)$ as the space-dependent permittivity and permeability, and $\overline{\bar{G}}\left(\mathbf{r}, \mathbf{r}^{\prime}, \omega\right)$ as the Green's tensor for a point dipole near a surface $[31,32]$. The Green's tensor is defined as the solution to the Helmholtz equation in the presence of the boundary conditions

$$
\begin{aligned}
& \nabla \times \nabla \times \overline{\bar{G}}\left(\mathbf{r}, \mathbf{r}^{\prime}, \omega\right)-\frac{\omega^{2}}{c^{2}} \epsilon(\mathbf{r}, \omega) \mu(\mathbf{r}, \omega) \overline{\bar{G}}\left(\mathbf{r}, \mathbf{r}^{\prime}, \omega\right) \\
& =\delta\left(\mathbf{r}-\mathbf{r}^{\prime}\right) \rrbracket .
\end{aligned}
$$

The total Green's tensor can be expressed as

$$
\overline{\bar{G}}\left(\mathbf{r}_{1}, \mathbf{r}_{2}, \omega\right)=\overline{\bar{G}}_{\text {free }}\left(\mathbf{r}_{1}, \mathbf{r}_{2}, \omega\right)+\overline{\bar{G}}_{\text {sc }}\left(\mathbf{r}_{1}, \mathbf{r}_{2}, \omega\right),
$$

where $\overline{\bar{G}}_{\text {free }}\left(\mathbf{r}_{1}, \mathbf{r}_{2}, \omega\right)$ and $\overline{\bar{G}}_{\text {sc }}\left(\mathbf{r}_{1}, \mathbf{r}_{2}, \omega\right)$ refer to the freespace and scattering components of the total Green's tensor. 


\section{APPENDIX B: SCATTERING GREEN'S TENSOR NEAR A PLANAR SURFACE}

For a point dipole located at the position $\mathbf{r}_{1}$ near an infinite planar half space, one can write the scattering Green's tensor as [31]

$$
\left.\begin{array}{rl}
\overline{\bar{G}}_{\mathrm{sc}}\left(\mathbf{r}_{1}, \mathbf{r}_{2}, \omega\right)= & \frac{1}{8 \pi} \int_{0}^{\infty} d k_{\|} \frac{k_{\|}}{\kappa_{\perp}} e^{-\kappa_{\perp}\left(z_{1}+z_{2}\right)}\left[\left(\begin{array}{ccc}
J_{0}\left(k_{\|} x_{12}\right)+J_{2}\left(k_{\|} x_{12}\right) & 0 & 0 \\
0 & J_{0}\left(k_{\|} x_{12}\right)-J_{2}\left(k_{\|} x_{12}\right) & 0 \\
0 & 0 & 0
\end{array}\right) r_{s}\right. \\
& +\frac{c^{2}}{\omega^{2}}\left(\begin{array}{ccc}
\kappa_{\perp}^{2}\left[J_{0}\left(k_{\|} x_{12}\right)-J_{2}\left(k_{\|} x_{12}\right)\right] & 2 k_{\|} \kappa_{\perp} J_{1}\left(k_{\|} x_{12}\right) \\
0 & \kappa_{\perp}^{2}\left[J_{0}\left(k_{\|} x_{12}\right)+J_{2}\left(k_{\|} x_{12}\right)\right] & 0 \\
-2 k_{\|} \kappa_{\perp} J_{1}\left(k_{\|} x_{12}\right) & 0 & 2 k_{\|}^{2} J_{0}\left(k_{\|} x_{12}\right)
\end{array}\right) r_{p}
\end{array}\right],
$$

with $\left|\mathbf{r}_{1}-\mathbf{r}_{2}\right|=r,\left(\mathbf{r}_{1}+\mathbf{r}_{2}\right) \cdot \mathbf{e}_{z}=\left(z_{1}+z_{2}\right)$, and we have defined the relative coordinate vector between the points $\mathbf{r}_{1}$ and $\mathbf{r}_{2}$ as $\frac{\mathbf{r}_{1}-\mathbf{r}_{2}}{\left|\mathbf{r}_{1}-\mathbf{r}_{2}\right|} \equiv\left(\frac{x_{12}}{r}, 0, \frac{z_{1}-z_{2}}{r}\right)^{\mathrm{T}}$. Here, $r_{s, p}$ are the Fresnel reflection coefficients for the $s$ and $p$ polarizations reflecting off the surface, and $\kappa_{\perp}^{2}=-k^{2}+k_{\|}^{2}$, where $k=\omega / c$. Assuming that the medium can be treated as homogeneous and isotropic, and can be well described in terms of its bulk optical properties at the length scales of the particle-surface separations, we can consider that all the information about the surface material is accounted for in the following Fresnel reflection coefficients,

$$
\begin{aligned}
& r_{p}\left(\kappa_{\perp}, \omega\right)=\frac{\epsilon(\omega) \kappa_{\perp}-\sqrt{-[\epsilon(\omega) \mu(\omega)-1] k^{2}+\kappa_{\perp}^{2}}}{\epsilon(\omega) \kappa_{\perp}+\sqrt{-[\epsilon(\omega) \mu(\omega)-1] k^{2}+\kappa_{\perp}^{2}}}, \\
& r_{s}\left(\kappa_{\perp}, \omega\right)=\frac{\mu(\omega) \kappa_{\perp}-\sqrt{-[\epsilon(\omega) \mu(\omega)-1] k^{2}+\kappa_{\perp}^{2}}}{\mu(\omega) \kappa_{\perp}+\sqrt{-[\epsilon(\omega) \mu(\omega)-1] k^{2}+\kappa_{\perp}^{2}}} .
\end{aligned}
$$

In the nonretarded limit $(\tilde{z} \ll 1)$, one can expand the Fresnel coefficients in Eq. (B2) to lowest order in $\left|\sqrt{\epsilon(\omega)-1} \omega /\left(\kappa_{\perp} c\right)\right|$ as

$$
\begin{gathered}
r_{p}\left(\kappa_{\perp}, \omega\right) \approx \frac{\epsilon(\omega)-1}{\epsilon(\omega)+1}+\frac{\epsilon(\omega)[\epsilon(\omega)-1]}{[\epsilon(\omega)+1]^{2}} \frac{\omega^{2}}{\kappa_{\perp}^{2} c^{2}}, \\
r_{s}\left(\kappa_{\perp}, \omega\right) \approx \frac{1}{4}[\epsilon(\omega)-1] \frac{\omega^{2}}{\kappa_{\perp}^{2} c^{2}} .
\end{gathered}
$$

For coincident points $\left(\mathbf{r}_{1}=\mathbf{r}_{2}=\mathbf{r}_{0}\right)$, one can write the scattering Green's tensor as [31]

$$
\overline{\bar{G}}_{\mathrm{sc}}\left(\mathbf{r}_{0}, \mathbf{r}_{0}, \omega\right)=\frac{1}{8 \pi} \int_{0}^{\infty} \frac{d k_{\|} k_{\|}}{\kappa_{\perp}} e^{-2 \kappa_{\perp} z}\left[r_{p}\left(\kappa_{\perp}, \omega\right) \frac{c^{2}}{\omega^{2}}\left(\begin{array}{ccc}
\kappa_{\perp}^{2} & 0 & 0 \\
0 & \kappa_{\perp}^{2} & 0 \\
0 & 0 & 2 k_{\|}^{2}
\end{array}\right)+r_{s}\left(\kappa_{\perp}, \omega\right)\left(\begin{array}{ccc}
1 & 0 & 0 \\
0 & 1 & 0 \\
0 & 0 & 0
\end{array}\right)\right] .
$$

The double $z$ derivative of the $x x$ component of the recoil Green's tensor [see Eq. (32)] is then given as

$$
\partial_{z} \operatorname{Im} \overline{\bar{G}}_{\mathrm{sc}}^{x x}\left(\mathbf{r}_{0}, \mathbf{r}_{0}, \omega_{0}\right) \partial_{z}=\frac{1}{8 \pi} \operatorname{Im}\left[\int_{0}^{\infty} \frac{d k_{\|} k_{\|}}{\kappa_{\perp}} \kappa_{\perp}^{2} e^{-2 \kappa_{\perp} z}\left\{r_{p}\left(\kappa_{\perp}, k_{0}\right) \frac{\kappa_{\perp}^{2}}{k_{0}^{2}}+r_{s}\left(\kappa_{\perp}, k_{0}\right)\right\}\right] .
$$

The free-space Green's tensor between the points $\mathbf{r}_{1}$ and $\mathbf{r}_{2}$ is given as

$$
\overline{\bar{G}}_{\text {free }}\left(\mathbf{r}_{1}, \mathbf{r}_{2}, \omega\right)=-\frac{e^{i k r}}{4 \pi k^{2} r^{3}}\left(\begin{array}{ccc}
f(k r)-h(k r) \frac{x_{12}^{2}}{r^{2}} & 0 & 0 \\
0 & f(k r) & 0 \\
0 & 0 & f(k r)
\end{array}\right) \text {, }
$$

where $f(x) \equiv 1-i x-x^{2}, h(x) \equiv 3-3 i x-x^{2}$.

It can be seen that the free-space recoil Green's tensor is given as the double $z$ derivative of the $x x$ component of as

$$
\partial_{z} \overline{\bar{G}}_{\text {free }}\left(\mathbf{r}_{0}, \mathbf{r}_{0}, \omega\right) \partial_{z}=\frac{\omega^{3}}{15 \pi c^{3}}
$$




\section{APPENDIX C: DERIVATION OF THE DRIVEN CASIMIR-POLDER POTENTIAL}

Using time-dependent second-order perturbation theory, we can define the energy correction and the modification to the dissipation rate of the system arising due to the driven CP Hamiltonian $\hat{H}_{\mathrm{DCP}}^{(0)}(t)$ [see Eq. (13)] as $U_{\mathrm{DCP}}(\mathbf{r})=\hbar \operatorname{Re} \chi$, and $\gamma_{\mathrm{sc}}(\mathbf{r})=$ $-\operatorname{Im} \chi$, where

$$
\begin{aligned}
\chi & =-\frac{i}{\hbar^{2}}\left\langle\int_{0}^{\infty} d \tau \tilde{H}_{\mathrm{DCP}}^{(0)}(t) \tilde{H}_{\mathrm{DCP}}^{(0)}(t-\tau)\right\rangle_{F} \\
& =-\frac{i}{\hbar^{2}}\left\langle\int_{0}^{\infty} d \tau\left\{\mathbf{P}_{0}(\mathbf{r}, t) \cdot \tilde{\mathbf{E}}_{f}(\mathbf{r}, t)+\tilde{\mathbf{P}}_{f}(\mathbf{r}, t) \cdot \mathbf{E}_{0}(\mathbf{r}, t)\right\}\left\{\mathbf{P}_{0}(\mathbf{r}, t-\tau) \cdot \tilde{\mathbf{E}}_{f}(\mathbf{r}, t-\tau)+\tilde{\mathbf{P}}_{f}(\mathbf{r}, t-\tau) \cdot \mathbf{E}_{0}(\mathbf{r}, t-\tau)\right\}\right\rangle_{F}
\end{aligned}
$$

where we have defined the electric field and polarization fluctuation operators in the interaction picture as $\tilde{\mathcal{O}}(t) \equiv$ $e^{-i\left(\hat{H}_{M}+\hat{H}_{F}\right) t} \hat{\mathcal{O}} e^{i\left(\hat{H}_{M}+\hat{H}_{F}\right) t}$ [45]. The average is taken over the thermal state of the field. We note that the shifts and decay rates are consistent with those derived via the second-order Born-Markov master equation [27].

We further divide the above into four separate terms as follows:

$$
\begin{gathered}
(\mathrm{I}) \equiv-\frac{i}{\hbar^{2}}\left\langle\int_{0}^{\infty} d \tau\left\{\mathbf{P}_{0}(\mathbf{r}, t) \cdot \tilde{\mathbf{E}}_{f}(\mathbf{r}, t)\right\}\left\{\mathbf{P}_{0}(\mathbf{r}, t-\tau) \cdot \tilde{\mathbf{E}}_{f}(\mathbf{r}, t-\tau)\right\}\right\rangle_{F}, \\
(\mathrm{II}) \equiv-\frac{i}{\hbar^{2}}\left\langle\int_{0}^{\infty} d \tau\left\{\mathbf{P}_{0}(\mathbf{r}, t) \cdot \tilde{\mathbf{E}}_{f}(\mathbf{r}, t)\right\}\left\{\tilde{\mathbf{P}}_{f}(\mathbf{r}, t-\tau) \cdot \mathbf{E}_{0}(\mathbf{r}, t-\tau)\right\}\right\rangle_{F}, \\
(\mathrm{III}) \equiv-\frac{i}{\hbar^{2}}\left\langle\int_{0}^{\infty} d \tau\left\{\tilde{\mathbf{P}}_{f}(\mathbf{r}, t) \cdot \mathbf{E}_{0}(\mathbf{r}, t)\right\}\left\{\mathbf{P}_{0}(\mathbf{r}, t-\tau) \cdot \tilde{\mathbf{E}}_{f}(\mathbf{r}, t-\tau)\right\}\right\rangle_{F}, \\
(\mathrm{IV}) \equiv-\frac{i}{\hbar^{2}}\left\langle\int_{0}^{\infty} d \tau\left\{\tilde{\mathbf{P}}_{f}(\mathbf{r}, t) \cdot \mathbf{E}_{0}(\mathbf{r}, t)\right\}\left\{\tilde{\mathbf{P}}_{f}(\mathbf{r}, t-\tau) \cdot \mathbf{E}_{0}(\mathbf{r}, t-\tau)\right\}\right\rangle_{F},
\end{gathered}
$$

such that $\chi=(\mathrm{I})+(\mathrm{II})+(\mathrm{III})+(\mathrm{IV})$. Let us consider the first term as follows:

$$
\begin{aligned}
(\mathrm{I})= & \frac{i}{\hbar^{2}} \operatorname{Tr}_{B}\left[\int_{0}^{\infty} d \tau\left\{\mathbf{P}_{0}(\mathbf{r}, t) \cdot \tilde{\mathbf{E}}_{f}(\mathbf{r}, t)\right\}\left\{\mathbf{P}_{0}(\mathbf{r}, t-\tau) \cdot \tilde{\mathbf{E}}_{f}(\mathbf{r}, t-\tau)\right\} \hat{\rho}_{F}\right] \\
= & -\frac{i}{4 \hbar^{2}} \operatorname{Tr}_{B}\left[\int_{0}^{\infty} d \tau\left\{\alpha\left(\omega_{0}\right) \mathcal{E}_{0}(\mathbf{r}) e^{-i \omega_{0} t}+\alpha\left(\omega_{0}\right) \mathcal{E}_{0}^{*}(\mathbf{r}) e^{i \omega_{0} t}\right\}\right. \\
& \cdot\left\{\int d \omega_{1} \sum_{\lambda_{1}=e, m} \int d^{3} r_{1}\left[\overline{\bar{G}}_{\lambda_{1}}\left(\mathbf{r}, \mathbf{r}_{1}, \omega_{1}\right) \cdot \hat{\mathbf{f}}_{\lambda_{1}}\left(\mathbf{r}_{1}, \omega_{1}\right) e^{-i \omega_{1} t}+\hat{\mathbf{f}}_{\lambda_{1}}^{\dagger}\left(\mathbf{r}_{1}, \omega_{1}\right) \cdot \overline{\bar{G}}_{\lambda_{1}}^{\dagger}\left(\mathbf{r}, \mathbf{r}_{1}, \omega_{1}\right) e^{i \omega_{1} t}\right]\right\} \\
& \left\{\int d \omega_{2} \sum_{\lambda_{2}=e, m} \int d^{3} r_{2}\left[\overline{\bar{G}}_{\lambda_{2}}\left(\mathbf{r}, \mathbf{r}_{2}, \omega_{1}\right) \cdot \hat{\mathbf{f}}_{\lambda_{2}}\left(\mathbf{r}_{2}, \omega_{2}\right) e^{-i \omega_{2}(t-\tau)}+\hat{\mathbf{f}}_{\lambda_{2}}^{\dagger}\left(\mathbf{r}_{2}, \omega_{2}\right) \cdot \overline{\bar{G}}_{\lambda_{2}}^{\dagger}\left(\mathbf{r}, \mathbf{r}_{2}, \omega_{2}\right) e^{i \omega_{2}(t-\tau)}\right]\right\} \\
& \left.\cdot\left\{\alpha\left(\omega_{0}\right) \mathcal{E}_{0}(\mathbf{r}) e^{-i \omega_{0}(t-\tau)}+\alpha\left(\omega_{0}\right) \mathcal{E}_{0}^{*}(\mathbf{r}) e^{i \omega_{0}(t-\tau)}\right\} \hat{\rho}_{F}\right] \\
=- & \frac{i}{4 \hbar^{2}} \int_{0}^{\infty} d \tau\left[\alpha\left(\omega_{0}\right)\right]^{2}\left[\mathcal{E}_{0}(\mathbf{r}) e^{-i \omega_{0} t}+\mathcal{E}_{0}^{*}(\mathbf{r}) e^{i \omega_{0} t}\right] \cdot\left[\int d \omega \sum_{\lambda=e, m} \int d^{3} r^{\prime}\left\{\overline{\bar{G}}_{\lambda}\left(\mathbf{r}, \mathbf{r}^{\prime}, \omega\right) \cdot \overline{\bar{G}}_{\lambda}^{\dagger}\left(\mathbf{r}, \mathbf{r}^{\prime}, \omega\right)\right\}\right. \\
& \left.\left\{\left[n_{\mathrm{th}}(\omega)+1\right] e^{-i \omega \tau}+n_{\mathrm{th}}(\omega) e^{i \omega \tau}\right\}\right] \cdot\left[\mathcal{E}_{0}(\mathbf{r}) e^{-i \omega_{0}(t-\tau)}+\mathcal{E}_{0}^{*}(\mathbf{r}) e^{i \omega_{0}(t-\tau)}\right],
\end{aligned}
$$

where in the second step we take an average over the field density matrix $\hat{\rho}_{F}=\hat{\rho}_{\text {th }}$, such that

$$
\operatorname{Tr}_{B}\left[\hat{\mathbf{f}}_{\lambda_{1}}^{\dagger}\left(\mathbf{r}_{1}, \omega_{1}\right) \cdot \hat{\mathbf{f}}_{\lambda_{2}}\left(\mathbf{r}_{2}, \omega_{2}\right) \rho_{F}\right]=n_{\mathrm{th}}\left(\omega_{1}\right) \delta_{\lambda_{1}, \lambda_{2}} \delta\left(\mathbf{r}_{1}-\mathbf{r}_{2}\right) \delta\left(\omega_{1}-\omega_{2}\right)
$$

Physically this is equivalent to saying that the virtual excitations of the EM field emitted and absorbed by the particle occur at the same frequency, position, and space coordinate, and have an average number expectation values of $n_{\text {th }}(\omega)$. We can further 
simplify (I) as

$$
\begin{aligned}
(\mathrm{I})= & -\frac{i}{4 \hbar^{2}} \int d \omega \frac{\mu_{0} \omega^{2}\left[\alpha\left(\omega_{0}\right)\right]^{2}}{\pi \hbar} \int_{0}^{\infty} d \tau\left[\mathcal{E}_{0}(\mathbf{r}) \cdot \operatorname{Im} \overline{\bar{G}}(\mathbf{r}, \mathbf{r}, \omega) \cdot \mathcal{E}_{0}^{*}(\mathbf{r}) e^{-i \omega_{0} \tau}\right. \\
& \left.+\mathcal{E}_{0}^{*}(\mathbf{r}) \cdot \operatorname{Im} \overline{\bar{G}}(\mathbf{r}, \mathbf{r}, \omega) \cdot \mathcal{E}_{0}(\mathbf{r}) e^{i \omega_{0} \tau}\left\{\left[n_{\mathrm{th}}(\omega)+1\right] e^{-i \omega \tau}+n_{\mathrm{th}}(\omega) e^{i \omega \tau}\right\}\right] \\
= & -i \frac{\left[2 n_{\mathrm{th}}\left(\omega_{0}\right)+1\right] \mu_{0} \omega_{0}^{2}\left[\alpha\left(\omega_{0}\right)\right]^{2}}{4 \hbar}\left[\mathcal{E}_{0}(\mathbf{r}) \cdot \operatorname{Im} \overline{\bar{G}}\left(\mathbf{r}, \mathbf{r}, \omega_{0}\right) \cdot \mathcal{E}_{0}^{*}(\mathbf{r})\right] \\
& -\frac{\left[2 n_{\mathrm{th}}\left(\omega_{0}\right)+1\right]\left[\alpha\left(\omega_{0}\right)\right]^{2}}{4 \hbar} \frac{\mu_{0} \omega_{0}^{2}}{2}\left[\mathcal{E}_{0}(\mathbf{r}) \cdot \operatorname{Re} \overline{\bar{G}}\left(\mathbf{r}, \mathbf{r}, \omega_{0}\right) \cdot \mathcal{E}_{0}^{*}(\mathbf{r})\right],
\end{aligned}
$$

where in the first step we have made the rotating-wave approximation and used the fluctuation-dissipation relation [31]

$$
\sum_{\lambda=e, m} \int d^{3} r^{\prime} \overline{\bar{G}}_{\lambda}\left(\mathbf{r}, \mathbf{r}^{\prime}, \omega\right) \overline{\bar{G}}_{\lambda}^{\dagger}\left(\mathbf{r}, \mathbf{r}^{\prime}, \omega\right)=\frac{\hbar \mu_{0} \omega^{2}}{\pi} \operatorname{Im} \overline{\bar{G}}(\mathbf{r}, \mathbf{r}, \omega) .
$$

In the second step while performing the integral over $\tau$, we note that $\int_{0}^{\infty} d \tau e^{i \tau x}=\pi \delta(x)+i \mathcal{P}\left(\frac{1}{x}\right)$. To evaluate the principal value term, we make a contour integral over the first and the second quadrants of the upper half complex plane.

We can similarly simplify the remaining terms to find that $(\mathrm{I})=(\mathrm{II})=(\mathrm{III})=(\mathrm{IV})$.

This yields the total potential as

$$
U_{\mathrm{DCP}}(\mathbf{r})=-\left[2 n_{\mathrm{th}}\left(\omega_{0}\right)+1\right] \frac{\mu_{0} \omega_{0}^{2}\left[\alpha\left(\omega_{0}\right)\right]^{2}}{2}\left[\mathcal{E}_{0}(\mathbf{r}) \cdot \operatorname{Re} \overline{\bar{G}}_{\mathrm{sc}}\left(\mathbf{r}, \mathbf{r}, \omega_{0}\right) \cdot \mathcal{E}_{0}^{*}(\mathbf{r})\right] .
$$

This is the driven CP potential as given in Eq. (14), which is in agreement with the result in Ref. [35] at zero temperature. We can similarly also find the surface-modified scattering rate as

$$
\gamma_{\mathrm{sc}}(\mathbf{r})=\frac{\mu_{0} \omega_{0}^{2}\left[\alpha\left(\omega_{0}\right)\right]^{2}}{\hbar}\left[2 n_{\mathrm{th}}\left(\omega_{0}\right)+1\right]\left[\mathcal{E}_{0}(\mathbf{r}) \cdot \operatorname{Im} \overline{\bar{G}}_{\mathrm{sc}}\left(\mathbf{r}, \mathbf{r}, \omega_{0}\right) \cdot \mathcal{E}_{0}^{*}(\mathbf{r})\right] .
$$

For a particle in the near-field limit of a surface with permittivity $\epsilon_{S}(\omega)$, for $n_{\text {th }}\left(\omega_{0}\right) \ll 1$ the above can be approximated as

$$
\gamma_{\mathrm{sc}}(\mathbf{r}) \approx \frac{\left[\alpha\left(\omega_{0}\right)\right]^{2}\left|\mathcal{E}_{0}\right|^{2}}{8 \pi \hbar \epsilon_{0} \tilde{z}^{3}} \operatorname{Im}\left[\frac{\epsilon_{S}\left(\omega_{0}\right)-1}{\epsilon_{S}\left(\omega_{0}\right)+1}\right] .
$$

\section{APPENDIX D: DERIVATION OF THE DISSIPATION AND NOISE KERNELS}

Let us consider the two-time correlation functions of the bath operators as follows,

$$
\begin{aligned}
\langle\tilde{\mathcal{B}}(t) \tilde{\mathcal{B}}(t-\tau)\rangle= & \left\langle\left[\mathbf{P}_{0}\left(\mathbf{r}_{0}, t\right) \cdot \frac{\partial}{\partial z} \tilde{\mathbf{E}}_{f}\left(\mathbf{r}_{0}, t\right)+\frac{\partial}{\partial z} \tilde{\mathbf{P}}_{f}\left(\mathbf{r}_{0}, t\right) \cdot \mathbf{E}_{0}\left(\mathbf{r}_{0}, t\right)+\frac{\partial}{\partial z} \mathbf{P}_{0}\left(\mathbf{r}_{0}, t\right) \cdot \tilde{\mathbf{E}}_{f}\left(\mathbf{r}_{0}\right)+\tilde{\mathbf{P}}_{f}\left(\mathbf{r}_{0}\right) \cdot \frac{\partial}{\partial z} \mathbf{E}_{0}\left(\mathbf{r}_{0}, t\right)\right]\right. \\
& \times\left[\mathbf{P}_{0}\left(\mathbf{r}_{0}, t-\tau\right) \cdot \frac{\partial}{\partial z} \tilde{\mathbf{E}}_{f}\left(\mathbf{r}_{0}, t-\tau\right)+\frac{\partial}{\partial z} \tilde{\mathbf{P}}_{f}\left(\mathbf{r}_{0}, t-\tau\right) \cdot \mathbf{E}_{0}\left(\mathbf{r}_{0}, t-\tau\right)\right. \\
& \left.\left.+\frac{\partial}{\partial z} \mathbf{P}_{0}\left(\mathbf{r}_{0}, t-\tau\right) \cdot \tilde{\mathbf{E}}_{f}\left(\mathbf{r}_{0}, t-\tau\right)+\tilde{\mathbf{P}}_{f}\left(\mathbf{r}_{0}, t-\tau\right) \cdot \frac{\partial}{\partial z} \mathbf{E}_{0}\left(\mathbf{r}_{0}, t-\tau\right)\right]\right) .
\end{aligned}
$$

We further divide the correlator above into 16 parts as follows:

$$
\begin{aligned}
C_{1}(\tau) & \equiv\left\langle\left[\mathbf{P}_{0}\left(\mathbf{r}_{0}, t\right) \cdot \frac{\partial}{\partial z} \tilde{\mathbf{E}}_{f}\left(\mathbf{r}_{0}, t\right)\right]\left[\mathbf{P}_{0}\left(\mathbf{r}_{0}, t-\tau\right) \cdot \frac{\partial}{\partial z} \tilde{\mathbf{E}}_{f}\left(\mathbf{r}_{0}, t-\tau\right)\right]\right\rangle, \\
C_{2}(\tau) & \equiv\left\langle\left[\mathbf{P}_{0}\left(\mathbf{r}_{0}, t\right) \cdot \frac{\partial}{\partial z} \tilde{\mathbf{E}}_{f}\left(\mathbf{r}_{0}, t\right)\right]\left[\frac{\partial}{\partial z} \tilde{\mathbf{P}}_{f}\left(\mathbf{r}_{0}, t-\tau\right) \cdot \mathbf{E}_{0}\left(\mathbf{r}_{0}, t-\tau\right)\right]\right\rangle, \\
C_{3}(\tau) & \equiv\left\langle\left[\frac{\partial}{\partial z} \tilde{\mathbf{P}}_{f}\left(\mathbf{r}_{0}, t\right) \cdot \mathbf{E}_{0}\left(\mathbf{r}_{0}, t\right)\right]\left[\mathbf{P}_{0}\left(\mathbf{r}_{0}, t-\tau\right) \cdot \frac{\partial}{\partial z} \tilde{\mathbf{E}}_{f}\left(\mathbf{r}_{0}, t-\tau\right)\right]\right\rangle, \\
C_{4}(\tau) & \equiv\left\langle\left[\frac{\partial}{\partial z} \tilde{\mathbf{P}}_{f}\left(\mathbf{r}_{0}, t\right) \cdot \mathbf{E}_{0}\left(\mathbf{r}_{0}, t\right)\right]\left[\frac{\partial}{\partial z} \tilde{\mathbf{P}}_{f}\left(\mathbf{r}_{0}, t-\tau\right) \cdot \mathbf{E}_{0}\left(\mathbf{r}_{0}, t-\tau\right)\right]\right\rangle, \\
C_{5}(\tau) & \equiv\left\langle\left[\frac{\partial}{\partial z} \mathbf{P}_{0}\left(\mathbf{r}_{0}, t\right) \cdot \tilde{\mathbf{E}}_{f}\left(\mathbf{r}_{0}, t\right)\right]\left[\frac{\partial}{\partial z} \mathbf{P}_{0}\left(\mathbf{r}_{0}, t-\tau\right) \cdot \tilde{\mathbf{E}}_{f}\left(\mathbf{r}_{0}, t-\tau\right)\right]\right\rangle, \\
C_{6}(\tau) & \equiv\left\langle\left[\frac{\partial}{\partial z} \mathbf{P}_{0}\left(\mathbf{r}_{0}, t\right) \cdot \tilde{\mathbf{E}}_{f}\left(\mathbf{r}_{0}, t\right)\right]\left[\tilde{\mathbf{P}}_{f}\left(\mathbf{r}_{0}, t-\tau\right) \cdot \frac{\partial}{\partial z} \mathbf{E}_{0}\left(\mathbf{r}_{0}, t-\tau\right)\right]\right\rangle,
\end{aligned}
$$




$$
\begin{aligned}
& C_{7}(\tau) \equiv\left\langle\left[\tilde{\mathbf{P}}_{f}\left(\mathbf{r}_{0}, t\right) \cdot \frac{\partial}{\partial z} \mathbf{E}_{0}\left(\mathbf{r}_{0}, t\right)\right]\left[\frac{\partial}{\partial z} \mathbf{P}_{0}\left(\mathbf{r}_{0}, t-\tau\right) \cdot \tilde{\mathbf{E}}_{f}\left(\mathbf{r}_{0}, t-\tau\right)\right]\right\rangle, \\
& C_{8}(\tau) \equiv\left\langle\left[\tilde{\mathbf{P}}_{f}\left(\mathbf{r}_{0}, t\right) \cdot \frac{\partial}{\partial z} \mathbf{E}_{0}\left(\mathbf{r}_{0}, t\right)\right]\left[\tilde{\mathbf{P}}_{f}\left(\mathbf{r}_{0}, t-\tau\right) \cdot \frac{\partial}{\partial z} \mathbf{E}_{0}\left(\mathbf{r}_{0}, t-\tau\right)\right]\right\rangle, \\
& C_{9}(\tau) \equiv\left\langle\left[\mathbf{P}_{0}\left(\mathbf{r}_{0}, t\right) \cdot \frac{\partial}{\partial z} \tilde{\mathbf{E}}_{f}\left(\mathbf{r}_{0}, t\right)\right]\left[\frac{\partial}{\partial z} \mathbf{P}_{0}\left(\mathbf{r}_{0}, t-\tau\right) \cdot \tilde{\mathbf{E}}_{f}\left(\mathbf{r}_{0}, t-\tau\right)\right]\right\rangle, \\
& C_{10}(\tau) \equiv\left\langle\left[\mathbf{P}_{0}\left(\mathbf{r}_{0}, t\right) \cdot \frac{\partial}{\partial z} \tilde{\mathbf{E}}_{f}\left(\mathbf{r}_{0}, t\right)\right]\left[\tilde{\mathbf{P}}_{f}\left(\mathbf{r}_{0}, t-\tau\right) \cdot \frac{\partial}{\partial z} \mathbf{E}_{0}\left(\mathbf{r}_{0}, t-\tau\right)\right]\right\rangle, \\
& C_{11}(\tau) \equiv\left\langle\left[\frac{\partial}{\partial z} \tilde{\mathbf{P}}_{f}\left(\mathbf{r}_{0}, t\right) \cdot \mathbf{E}_{0}\left(\mathbf{r}_{0}, t\right)\right]\left[\frac{\partial}{\partial z} \mathbf{P}_{0}\left(\mathbf{r}_{0}, t-\tau\right) \cdot \tilde{\mathbf{E}}_{f}\left(\mathbf{r}_{0}, t-\tau\right)\right]\right\rangle, \\
& C_{12}(\tau) \equiv\left\langle\left[\frac{\partial}{\partial z} \tilde{\mathbf{P}}_{f}\left(\mathbf{r}_{0}, t\right) \cdot \mathbf{E}_{0}\left(\mathbf{r}_{0}, t\right)\right]\left[\tilde{\mathbf{P}}_{f}\left(\mathbf{r}_{0}, t-\tau\right) \cdot \frac{\partial}{\partial z} \mathbf{E}_{0}\left(\mathbf{r}_{0}, t-\tau\right)\right]\right\rangle, \\
& C_{13}(\tau) \equiv\left\langle\left[\frac{\partial}{\partial z} \mathbf{P}_{0}\left(\mathbf{r}_{0}, t\right) \cdot \tilde{\mathbf{E}}_{f}\left(\mathbf{r}_{0}, t\right)\right]\left[\mathbf{P}_{0}\left(\mathbf{r}_{0}, t-\tau\right) \cdot \frac{\partial}{\partial z} \tilde{\mathbf{E}}_{f}\left(\mathbf{r}_{0}, t-\tau\right)\right]\right\rangle, \\
& C_{14}(\tau) \equiv\left\langle\left[\frac{\partial}{\partial z} \mathbf{P}_{0}\left(\mathbf{r}_{0}, t\right) \cdot \tilde{\mathbf{E}}_{f}\left(\mathbf{r}_{0}, t\right)\right]\left[\frac{\partial}{\partial z} \tilde{\mathbf{P}}_{f}\left(\mathbf{r}_{0}, t-\tau\right) \cdot \mathbf{E}_{0}\left(\mathbf{r}_{0}, t-\tau\right)\right]\right\rangle, \\
& C_{15}(\tau) \equiv\left\langle\left[\tilde{\mathbf{P}}_{f}\left(\mathbf{r}_{0}, t\right) \cdot \frac{\partial}{\partial z} \mathbf{E}_{0}\left(\mathbf{r}_{0}, t\right)\right]\left[\mathbf{P}_{0}\left(\mathbf{r}_{0}, t-\tau\right) \cdot \frac{\partial}{\partial z} \tilde{\mathbf{E}}_{f}\left(\mathbf{r}_{0}, t-\tau\right)\right]\right\rangle, \\
& C_{16}(\tau) \equiv\left\langle\left[\tilde{\mathbf{P}}_{f}\left(\mathbf{r}_{0}, t\right) \cdot \frac{\partial}{\partial z} \mathbf{E}_{0}\left(\mathbf{r}_{0}, t\right)\right]\left[\frac{\partial}{\partial z} \tilde{\mathbf{P}}_{f}\left(\mathbf{r}_{0}, t-\tau\right) \cdot \mathbf{E}_{0}\left(\mathbf{r}_{0}, t-\tau\right)\right]\right\rangle,
\end{aligned}
$$

such that $\langle\tilde{\mathcal{B}}(t) \tilde{\mathcal{B}}(t-\tau)\rangle=\sum_{j=1}^{16} C_{j}(\tau)$.

We now consider the first term closely as follows:

$$
\begin{aligned}
& C_{1}(\tau)=\left\langle\left[\mathbf{P}_{0}\left(\mathbf{r}_{0}, t\right) \cdot \frac{\partial}{\partial z} \tilde{\mathbf{E}}_{f}\left(\mathbf{r}_{0}, t\right)\right]\left[\mathbf{P}_{0}\left(\mathbf{r}_{0}, t-\tau\right) \cdot \frac{\partial}{\partial z} \tilde{\mathbf{E}}_{f}\left(\mathbf{r}_{0}, t-\tau\right)\right]\right\rangle \\
& =\frac{1}{4}\left\langle\left[\alpha\left(\omega_{0}\right) \mathcal{E}_{0}\left(\mathbf{r}_{0}\right) e^{-i \omega_{0} t}+\alpha\left(\omega_{0}\right) \mathcal{E}_{0}^{*}\left(\mathbf{r}_{0}\right) e^{i \omega_{0} t}\right]\right. \\
& \cdot\left[\int d \omega_{1} \sum_{\lambda_{1}=e, m} \int d^{3} r_{1}\left\{\frac{\partial}{\partial z} \overline{\bar{G}}_{\lambda_{1}}\left(\mathbf{r}_{0}, \mathbf{r}_{1}, \omega_{1}\right) \cdot \hat{\mathbf{f}}_{\lambda_{1}}\left(\mathbf{r}_{1}, \omega_{1}\right) e^{-i \omega_{1} t}+\hat{\mathbf{f}}_{\lambda_{1}}^{\dagger}\left(\mathbf{r}_{1}, \omega_{1}\right) \cdot \frac{\partial}{\partial z} \overline{\bar{G}}_{\lambda_{1}}^{\dagger}\left(\mathbf{r}_{0}, \mathbf{r}_{1}, \omega_{1}\right) e^{i \omega_{1} t}\right\}\right] \\
& {\left[\int d \omega_{2} \sum_{\lambda_{2}=e, m} \int d^{3} r_{2}\left\{\frac{\partial}{\partial z} \overline{\bar{G}}_{\lambda_{2}}\left(\mathbf{r}_{0}, \mathbf{r}_{2}, \omega_{1}\right) \cdot \hat{\mathbf{f}}_{\lambda_{2}}\left(\mathbf{r}_{2}, \omega_{2}\right) e^{-i \omega_{2}(t-\tau)}+\hat{\mathbf{f}}_{\lambda_{2}}^{\dagger}\left(\mathbf{r}_{2}, \omega_{2}\right) \cdot \frac{\partial}{\partial z} \overline{\bar{G}}_{\lambda_{2}}^{\dagger}\left(\mathbf{r}_{0}, \mathbf{r}_{2}, \omega_{2}\right) e^{i \omega_{2}(t-\tau)}\right\}\right]} \\
& \left.\cdot\left[\alpha\left(\omega_{0}\right) \mathcal{E}_{0}\left(\mathbf{r}_{0}\right) e^{-i \omega_{0}(t-\tau)}+\alpha\left(\omega_{0}\right) \mathcal{E}_{0}^{*}\left(\mathbf{r}_{0}\right) e^{i \omega_{0}(t-\tau)}\right]\right), \\
& =\frac{\mu_{0} \hbar}{2 \pi} \cos \left(\omega_{0} \tau\right) \int d \omega \omega^{2}\left(\alpha\left(\omega_{0}\right)\right)^{2}\left[\mathcal{E}_{0}\left(\mathbf{r}_{0}\right) \cdot \partial_{z} \operatorname{Im} \overline{\bar{G}}\left(\mathbf{r}_{0}, \mathbf{r}_{0}, \omega\right) \partial_{z} \cdot \mathcal{E}_{0}^{*}\left(\mathbf{r}_{0}\right)\right]\left[n_{\mathrm{th}}(\omega) e^{i \omega \tau}+\left\{n_{\mathrm{th}}(\omega)+1\right\} e^{-i \omega \tau}\right],
\end{aligned}
$$

where we have averaged over the thermal state of the field and used the fluctuation-dissipation relation for the Green's tensor in obtaining the third step.

Similarly we obtain

$$
\begin{gathered}
C_{2}(\tau)=\frac{\mu_{0} \hbar}{2 \pi} \cos \left(\omega_{0} \tau\right) \int d \omega \omega^{2} \alpha\left(\omega_{0}\right) \alpha(\omega)\left[\mathcal{E}_{0}\left(\mathbf{r}_{0}\right) \cdot \partial_{z} \operatorname{Im} \overline{\bar{G}}\left(\mathbf{r}_{0}, \mathbf{r}_{0}, \omega\right) \partial_{z} \cdot \mathcal{E}_{0}^{*}\left(\mathbf{r}_{0}\right)\right]\left[n_{\mathrm{th}}(\omega) e^{i \omega \tau}+\left\{n_{\mathrm{th}}(\omega)+1\right\} e^{-i \omega \tau},\right] \\
C_{3}(\tau)=\frac{\mu_{0} \hbar}{2 \pi} \cos \left(\omega_{0} \tau\right) \int d \omega \omega^{2} \alpha\left(\omega_{0}\right) \alpha(\omega)\left[\mathcal{E}_{0}\left(\mathbf{r}_{0}\right) \cdot \partial_{z} \operatorname{Im} \overline{\bar{G}}\left(\mathbf{r}_{0}, \mathbf{r}_{0}, \omega\right) \partial_{z} \cdot \mathcal{E}_{0}^{*}\left(\mathbf{r}_{0}\right)\right]\left[n_{\mathrm{th}}(\omega) e^{i \omega \tau}+\left\{n_{\mathrm{th}}(\omega)+1\right\} e^{-i \omega \tau},\right] \\
C_{4}(\tau)=\frac{\mu_{0} \hbar}{2 \pi} \cos \left(\omega_{0} \tau\right) \int d \omega \omega^{2}[\alpha(\omega)]^{2}\left[\mathcal{E}_{0}\left(\mathbf{r}_{0}\right) \cdot \partial_{z} \operatorname{Im} \overline{\bar{G}}\left(\mathbf{r}_{0}, \mathbf{r}_{0}, \omega\right) \partial_{z} \cdot \mathcal{E}_{0}^{*}\left(\mathbf{r}_{0}\right)\right]\left[n_{\mathrm{th}}(\omega) e^{i \omega \tau}+\left\{n_{\mathrm{th}}(\omega)+1\right\} e^{-i \omega \tau} \cdot\right]
\end{gathered}
$$

Summing together Eqs. (D19)-(D23), we get

$$
\sum_{j=1}^{4} C_{j}(\tau)=\frac{\mu_{0} \hbar}{2 \pi} \cos \left(\omega_{0} \tau\right) \int d \omega \omega^{2}\left[\alpha\left(\omega_{0}\right)+\alpha(\omega)\right]^{2}\left[\mathcal{E}_{0}\left(\mathbf{r}_{0}\right) \cdot\left\{\partial_{z} \operatorname{Im} \overline{\bar{G}}\left(\mathbf{r}_{0}, \mathbf{r}_{0}, \omega\right) \partial_{z}\right\} \cdot \mathcal{E}_{0}^{*}\left(\mathbf{r}_{0}\right)\right]\left[\left\{n_{\mathrm{th}}(\omega)+1\right\} e^{-i \omega \tau}+n_{\mathrm{th}}(\omega) e^{i \omega \tau}\right]
$$


Similarly, it can be shown that

$$
\begin{aligned}
& \sum_{j=5}^{8} C_{j}(\tau)=\frac{\mu_{0} \hbar}{2 \pi} \cos \left(\omega_{0} \tau\right) \int d \omega \omega^{2}\left[\alpha\left(\omega_{0}\right)+\alpha(\omega)\right]^{2}\left[\partial_{z} \mathcal{E}_{0}\left(\mathbf{r}_{0}\right) \cdot \operatorname{Im} \overline{\bar{G}}\left(\mathbf{r}_{0}, \mathbf{r}_{0}, \omega\right) \cdot \partial_{z} \mathcal{E}_{0}^{*}\left(\mathbf{r}_{0}\right)\right]\left[\left\{n_{\mathrm{th}}(\omega)+1\right\} e^{-i \omega \tau}+n_{\mathrm{th}}(\omega) e^{i \omega \tau}\right], \\
& \sum_{j=9}^{12} C_{j}(\tau)=\frac{\mu_{0} \hbar}{2 \pi} \cos \left(\omega_{0} \tau\right) \int d \omega \omega^{2}\left[\alpha\left(\omega_{0}\right)+\alpha(\omega)\right]^{2}\left[\mathcal{E}_{0}\left(\mathbf{r}_{0}\right) \cdot\left\{\partial_{z} \operatorname{Im} \overline{\bar{G}}\left(\mathbf{r}_{0}, \mathbf{r}_{0}, \omega\right)\right\} \cdot \partial_{z} \mathcal{E}_{0}^{*}\left(\mathbf{r}_{0}\right)\right]\left[\left\{n_{\mathrm{th}}(\omega)+1\right\} e^{-i \omega \tau}+n_{\mathrm{th}}(\omega) e^{i \omega \tau}\right] \\
& \sum_{j=13}^{16} C_{j}(\tau)=\frac{\mu_{0} \hbar}{2 \pi} \cos \left(\omega_{0} \tau\right) \int d \omega \omega^{2}\left[\alpha\left(\omega_{0}\right)+\alpha(\omega)\right]^{2}\left[\partial_{z} \mathcal{E}_{0}\left(\mathbf{r}_{0}\right) \cdot\left\{\operatorname{Im} \overline{\bar{G}}\left(\mathbf{r}_{0}, \mathbf{r}_{0}, \omega\right) \partial_{z}\right\} \cdot \mathcal{E}_{0}^{*}\left(\mathbf{r}_{0}\right)\right]\left[\left\{n_{\mathrm{th}}(\omega)+1\right\} e^{-i \omega \tau}+n_{\mathrm{th}}(\omega) e^{i \omega \tau}\right] .
\end{aligned}
$$

Equations (D24)-(D27) yield

$$
\langle\tilde{\mathcal{B}}(t-\tau) \tilde{\mathcal{B}}(t)\rangle=\frac{\mu_{0} \hbar}{2 \pi} \cos \left(\omega_{0} \tau\right) \int d \omega \omega^{2}\left[\alpha\left(\omega_{0}\right)+\alpha(\omega)\right]^{2}\left[\left\{n_{\mathrm{th}}(\omega)+1\right\} e^{-i \omega \tau}+n_{\mathrm{th}}(\omega) e^{i \omega \tau}\right] g\left(\mathbf{r}_{0}, \omega\right),
$$

where we have defined

$$
\begin{aligned}
g\left(\mathbf{r}_{0}, \omega\right) \equiv & {\left[\mathcal{E}_{0}\left(\mathbf{r}_{0}\right) \cdot\left\{\partial_{z} \operatorname{Im} \overline{\bar{G}}\left(\mathbf{r}_{0}, \mathbf{r}_{0}, \omega\right) \partial_{z}\right\} \cdot \mathcal{E}_{0}^{*}\left(\mathbf{r}_{0}\right)+\partial_{z} \mathcal{E}_{0}\left(\mathbf{r}_{0}\right) \cdot \operatorname{Im} \overline{\bar{G}}\left(\mathbf{r}_{0}, \mathbf{r}_{0}, \omega\right) \cdot \partial_{z} \mathcal{E}_{0}^{*}\left(\mathbf{r}_{0}\right)\right.} \\
& \left.\mathcal{E}_{0}\left(\mathbf{r}_{0}\right) \cdot\left\{\partial_{z} \operatorname{Im} \overline{\bar{G}}\left(\mathbf{r}_{0}, \mathbf{r}_{0}, \omega\right)\right\} \cdot \partial_{z} \mathcal{E}_{0}^{*}\left(\mathbf{r}_{0}\right)+\partial_{z} \mathcal{E}_{0}\left(\mathbf{r}_{0}\right) \cdot\left\{\operatorname{Im} \overline{\bar{G}}\left(\mathbf{r}_{0}, \mathbf{r}_{0}, \omega\right) \partial_{z}\right\} \cdot \mathcal{E}_{0}^{*}\left(\mathbf{r}_{0}\right)\right]
\end{aligned}
$$

Similarly it can be shown

$$
\langle\tilde{\mathcal{B}}(t-\tau) \tilde{\mathcal{B}}(t)\rangle=\frac{\mu_{0} \hbar}{2 \pi} \cos \left(\omega_{0} \tau\right) \int d \omega \omega^{2}\left[\alpha\left(\omega_{0}\right)+\alpha(\omega)\right]^{2}\left[\left\{n_{\mathrm{th}}(\omega)+1\right\} e^{i \omega \tau}+n_{\mathrm{th}}(\omega) e^{-i \omega \tau}\right] g\left(\mathbf{r}_{0}, \omega\right) .
$$

Using (D28) and (D30) we can write the dissipation and noise kernels as

$$
\begin{gathered}
\mathcal{D}(\tau)=i[\langle\tilde{\mathcal{B}}(t) \tilde{\mathcal{B}}(t-\tau)\rangle-\langle\tilde{\mathcal{B}}(t-\tau) \tilde{\mathcal{B}}(t)\rangle] \\
=\frac{\mu_{0} \hbar}{\pi} \cos \left(\omega_{0} \tau\right) \int d \omega \omega^{2}\left[\alpha\left(\omega_{0}\right)+\alpha(\omega)\right]^{2} \sin (\omega \tau) g\left(\mathbf{r}_{0}, \omega\right), \\
\mathcal{N}(\tau)=\langle\tilde{\mathcal{B}}(t) \tilde{\mathcal{B}}(t-\tau)\rangle+\langle\tilde{\mathcal{B}}(t-\tau) \tilde{\mathcal{B}}(t)\rangle \\
=\frac{\mu_{0} \hbar}{\pi} \cos \left(\omega_{0} \tau\right) \int d \omega \omega^{2}\left[\alpha\left(\omega_{0}\right)+\alpha(\omega)\right]^{2}\left[2 n_{\mathrm{th}}(\omega)+1\right] \cos (\omega \tau) g\left(\mathbf{r}_{0}, \omega\right) .
\end{gathered}
$$

Further noting that $2 n_{\mathrm{th}}(\omega)+1=\operatorname{coth}\left(\frac{\hbar \omega}{2 k_{B} T}\right)$, we arrive at Eqs. (20) and (21), with the spectral density given by (22).

\section{APPENDIX E: DECOHERENCE FROM OTHER SOURCES}

As a reference, we compare the surface-fluctuationinduced decoherence with that due to other sources that can potentially be a limiting mechanism for preparing macroscopic quantum states as follows.

\section{Background gas scattering}

The decoherence rate due to background gas scattering [30] is given as

$$
\Lambda_{\text {gas }}=\frac{8}{3 \hbar^{2}} P_{\text {gas }}\left(2 \pi m_{\mathrm{gas}}\right)^{1 / 2} R^{2}\left(k_{B} T\right)^{1 / 2}
$$

where $P_{\text {gas }}$ is the gas pressure, and $m_{\text {gas }} \approx 5 \times 10^{-26} \mathrm{~kg}$ is the mass of a single gas molecule. For a background gas pressure of $P_{\text {gas }} \sim 1-10^{-11}$ mbar, one obtains a corresponding localization parameter $\Lambda_{\text {gas }} \sim 10^{33}-10^{20} \mathrm{~Hz} / \mathrm{m}^{2}$. One potential way to circumvent decoherence due to background gas scattering could be to perform the experiment on timescales shorter than those of average successive collisions of the system with gas molecules [25].

\section{Scattering of blackbody radiation}

The c.m. decoherence of the dielectric nanosphere induced due to scattering of blackbody radiation is given as [30]

$$
\Lambda_{\mathrm{BB}}=\frac{8 ! c}{18 \pi}\left[\frac{\alpha\left(\omega_{\mathrm{th}}\right)}{\pi \epsilon_{0}}\right]^{2}\left(\frac{k_{B} T}{\hbar c}\right)^{9} \zeta(9)
$$

where $\zeta(9) \approx 1.002$ refers to the Riemann $\zeta$ function, $\omega_{\text {th }} \equiv$ $\frac{2 \pi c T}{b}$ is the peak blackbody radiation frequency, with $b$ as the Wien's displacement constant. For $T \sim 1-100 \mathrm{~K}$, we find the blackbody-radiation-induced decoherence as $\Lambda_{\mathrm{BB}} \sim 10^{-7}$ $10^{11} \mathrm{~Hz} / \mathrm{m}^{2}$. We note that this corresponds to the decoherence arising from a purely thermal background, and for large 
enough temperatures can potentially exceed the decoherence from the driven CP interaction as we can see from Fig. 2(b).

\section{Laser noise}

The fluctuations of the laser intensity can result in the fluctuations of trap position which can in turn decohere the particle's c.m.. We first estimate the fluctuations in the trap position as a function of the laser intensity noise by considering that the trap position of the particle is given by $\partial_{z} U_{\text {Tot }}(\mathbf{r})=$ $\partial_{z}\left[U_{\mathrm{Tr}}(\mathbf{r})+U_{\mathrm{DCP}}(\mathbf{r})+U_{\mathrm{CP}}(\mathbf{r})\right]=0$. In the near-field regime the Casimir-Polder potential for a point particle can be written as [31]

$$
U_{\mathrm{CP}}(z) \approx \hbar \tilde{\Gamma}\left(\frac{V}{z^{3}}\right),
$$

where $\tilde{\Gamma} \approx \frac{1}{16 \pi^{2} \epsilon_{0}} \int_{0}^{\infty} d \xi \alpha(i \xi)\left(\frac{\epsilon_{S}(i \xi)+1}{\epsilon_{S}(i \xi)+1}\right)$. For a silica sphere near a dielectric half space $\tilde{\Gamma} \sim 10^{14} \mathrm{~Hz}$.
Considering that $U_{\mathrm{Tr}} \gg U_{\mathrm{DCP}}$, the fluctuations in trap position $z_{0}$ can be approximated as

$$
\delta z_{0} \approx \frac{3 \hbar \tilde{\Gamma} V}{M \Omega^{2} z_{0}^{4}}\left(\frac{\delta I}{I}\right) .
$$

We can estimate the resulting decoherence from a fluctuating trap position to be [46]

$$
\Lambda_{\text {Laser }}=\frac{M^{2} \Omega^{4}}{2 \hbar^{2}} S_{z z}(\Omega),
$$

where $S_{z z}(\Omega)=\int d \tau\langle\delta z(t+\tau) \delta z(t)\rangle e^{i \Omega \tau}$ is the position noise correlation function evaluated at the trap frequency.

For a standard Nd:YAG laser as used in Ref. [16], the relative intensity noise is roughly $150 \mathrm{~dB} / \mathrm{Hz}$, such that $\delta I / I \sim$ $10^{-15} \mathrm{~Hz}^{-1}$. Considering the trap position of the particle to be $z_{0} \sim 500 \mathrm{~nm}$, we get the corresponding position noise correlation $S_{z z} \sim 10^{-25} \mathrm{~m}^{2} / \mathrm{Hz}$. This yields a localization paramter of $\Lambda_{\text {Laser }} \sim 10^{31} \mathrm{~Hz} / \mathrm{m}^{2}$, which we note is comparable to the decoherence from background gas scattering.
[1] W. H. Zurek, Decoherence and the transition from quantum to classical, Phys. Today 44(10), 36 (1991).

[2] M. Bhattacharya, A. N. Vamivakas, and P. Barker, Levitated optomechanics: Introduction, J. Opt. Soc. Am. B 34, LO1 (2017).

[3] Z.-Q. Yin, A. A. Geraci, and T. Li, Optomechanics of Levitated dielectric particles, Int. J. Mod. Phys. B 27, 1330018 (2013).

[4] O. Romero-Isart, A. C. Pflanzer, M. L. Juan, R. Quidant, N. Kiesel, M. Aspelmeyer, and J. I. Cirac, Optically levitating dielectrics in the quantum regime: Theory and protocols, Phys. Rev. A 83, 013803 (2011).

[5] O. Romero-Isart, Quantum superposition of massive objects and collapse models, Phys. Rev. A 84, 052121 (2011).

[6] J. Bateman, S. Nimmrichter, K. Hornberger, and H. Ulbricht, Near-field interferometry of a free-falling nanoparticle from a point-like source, Nat. Commun. 5, 4788 (2014).

[7] U. Delić, M. Reisenbauer, D. Grass, N. Kiesel, V. Vuletić, and M. Aspelmeyer, Cavity Cooling of a Levitated Nanosphere by Coherent Scattering, Phys. Rev. Lett. 122, 123602 (2019).

[8] D. Windey, C. Gonzalez-Ballestero, P. Maurer, L. Novotny, O. Romero-Isart, and R. Reimann, Cavity-Based 3D Cooling of a Levitated Nanoparticle via Coherent Scattering, Phys. Rev. Lett. 122, 123601 (2019).

[9] F. Tebbenjohanns, M. Frimmer, A. Militaru, V. Jain, and L. Novotny, Cold Damping of an Optically Levitated Nanoparticle to Microkelvin Temperatures, Phys. Rev. Lett. 122, 223601 (2019).

[10] J. Ahn, Z. Xu, J. Bang, Y.-H. Deng, T. M. Hoang, Q. Han, R.M. Ma, and T. Li, Optically Levitated Nanodumbbell Torsion Balance and GHz Nanomechanical Rotor, Phys. Rev. Lett. 121, 033603 (2018).

[11] R. Reimann, M. Doderer, E. Hebestreit, R. Diehl, M. Frimmer, D. Windey, F. Tebbenjohanns, and L. Novotny, GHz Rotation of an Optically Trapped Nanoparticle in Vacuum, Phys. Rev. Lett. 121, 033602 (2018).

[12] S. Kuhn, B. A. Stickler, A. Kosloff, F. Patolsky, K. Hornberger, M. Arndt, and J. Millen, Optically driven ultra-stable nanomechanical rotor, Nat. Commun. 8, 1670 (2017).
[13] R. Diehl, E. Hebestreit, R. Reimann, F. Tebbenjohanns, M. Frimmer, and L. Novotny, Optical levitation and feedback cooling of a nanoparticle at subwavelength distances from a membrane, Phys. Rev. A 98, 013851 (2018).

[14] J. D. Thompson, T. G. Tiecke, N. P. de Leon, J. Feist, A. V. Akimov, M. Gullans, A. S. Zibrov, V. Vuletić, and M. D. Lukin, Coupling a single trapped atom to a nanoscale optical cavity, Science 340, 1202 (2013).

[15] M. L. Juan, G. Molina-Terriza, T. Volz, and O. RomeroIsart, Near-field levitated quantum optomechanics with nanodiamonds, Phys. Rev. A 94, 023841 (2016).

[16] L. Magrini, R. A. Norte, R. Riedinger, I. Marinković, D. Grass, U. Delić, S. Gröblacher, S. Hong, and M. Aspelmeyer, Nanophotonic near-field levitated optomechanics, Optica 5, 1597 (2018).

[17] A. I. Volokitin and B. N. J. Persson, Near-field radiative heat transfer and noncontact friction, Rev. Mod. Phys. 79, 1291 (2007).

[18] S. Scheel, P. K. Rekdal, P. L. Knight, and E. A. Hinds, Atomic spin decoherence near conducting and superconducting films, Phys. Rev. A 72, 042901 (2005).

[19] P. K. Rekdal, S. Scheel, P. L. Knight, and E. A. Hinds, Thermal spin flips in atom chips, Phys. Rev. A 70, 013811 (2004).

[20] B.-S. K. Skagerstam, U. Hohenester, A. Eiguren, and P. K. Rekdal, Spin Decoherence in Superconducting Atom Chips, Phys. Rev. Lett. 97, 070401 (2006).

[21] E. A. Hinds and I. G. Hughes, Magnetic atom optics: Mirrors, guides, traps, and chips for atoms, J. Phys. D: Appl. Phys. 32, R119 (1999).

[22] T. Schumm, S. Hofferberth, L. M. Andersson, S. Wildermuth, S. Groth, I. Bar-Joseph, J. Schmiedmayer and P. Krüger, Matterwave interferometry in a double well on an atom chip, Nat. Phys. 1, 57 (2005).

[23] Y.-J. Lin, I. Teper, C. Chin, and V. Vuletić, Impact of the Casimir-Polder Potential and Johnson Noise on Bose-Einstein Condensate Stability Near Surfaces, Phys. Rev. Lett. 92, 050404 (2004). 
[24] M. P. A. Jones, C. J. Vale, D. Sahagun, B. V. Hall, and E. A. Hinds, Spin Coupling between Cold Atoms and the Thermal Fluctuations of a Metal Surface, Phys. Rev. Lett. 91, 080401 (2003).

[25] H. Pino, J. Prat-Camps, K. Sinha, B. P. Venkatesh, and O. Romero-Isart, On-chip quantum interference of a superconducting microsphere, Quantum Sci. Technol. 3, 025001 (2018).

[26] B. L. Hu, J. P. Paz, and Y. Zhang, Quantum Brownian motion in a general environment: Exact master equation with nonlocal dissipation and colored noise, Phys. Rev. D 45, 2843 (1992).

[27] H.-P. Breuer and F. Petruccione, Theory of Open Quantum Systems (Oxford University Press, New York, 2002).

[28] F. C. Lombardo and P. I. Villar, Decoherence induced by zeropoint fluctuations in quantum Brownian motion, Phys. Lett. A 336, 16 (2004).

[29] E. Joos, and H. D. Zeh, The emergence of classical properties through interaction with the environment, Z. Phys. B: Condens. Matter 59, 223 (1985).

[30] M. Schlosshauer, Decoherence and the Quantum-to-Classical Transition (Springer, Berlin, 2007).

[31] S. Y. Buhmann, Dispersion Forces I (Springer, Berlin, 2012).

[32] S. Y. Buhmann, Dispersion Forces II (Springer, Berlin, 2012).

[33] T. Gruner and D. G. Welsch, Green-function approach to the radiation-field quantization for homogeneous and inhomogeneous Kramers-Kronig dielectrics, Phys. Rev. A 53, 1818 (1996).

[34] J. D. Jackson, Classical Electrodynamics (Wiley, New York, 1999).

[35] S. Fuchs, R. Bennett, R. V. Krems, and S. Y. Buhmann, Nonadditivity of Optical and Casimir-Polder Potentials, Phys. Rev. Lett. 121, 083603 (2018).
[36] D. E. Chang, K. Sinha, J. M. Taylor, and H. J. Kimble, Trapping atoms using nanoscale quantum vacuum forces, Nat. Commun. 5, 4343 (2014).

[37] C.-H. Wu, C.-I. Kuo, and L. H. Ford, Fluctuations of the retarded van der Waals force, Phys. Rev. A 65, 062102 (2002).

[38] M. Aspelmeyer, T. J. Kippenberg, and F. Marquardt, Cavity optomechanics, Rev. Mod. Phys. 86, 1391 (2014).

[39] J. L. Hemmerich, R. Bennett, T. Reisinger, S. Nimmrichter, J. Fiedler, H. Hahn, H. Gleiter, and S. Y. Buhmann, Impact of Casimir-Polder interaction on Poisson-spot diffraction at a dielectric sphere, Phys. Rev. A 94, 023621 (2016).

[40] I. Pirozhenko, A. Lambrecht, and V. B. Svetovoy, Sample dependence of the Casimir force, New J. Phys. 8, 238 (2006).

[41] A. W. Rodriguez, F. Capasso, and S. G. Johnson, The Casimir effect in microstructured geometries, Nat. Photonics 5, 211 (2011).

[42] L. M. Woods, D. A. R. Dalvit, A. Tkatchenko, P. RodriguezLopez, A. W. Rodriguez, and R. Podgornik, Materials perspective on Casimir and van der Waals interactions, Rev. Mod. Phys. 88, 045003 (2016).

[43] K. Sinha, Repulsive vacuum-induced forces on a magnetic particle, Phys. Rev. A 97, 032513 (2018).

[44] K. Sinha, B. P. Venkatesh, and P. Meystre, Collective Effects in Casimir-Polder Forces, Phys. Rev. Lett. 121, 183605 (2018).

[45] J. J. Sakurai, Modern Quantum Mechanics (Addison-Wesley, Boston, 2011).

[46] C. Henkel, S. Pötting, and M. Wilkens, Loss and heating of particles in small and noisy traps, Appl. Phys. B 69, 379 (1999). 